\title{
An XMM-Newton Study of the Mixed-morphology Supernova Remnant G346.6-0.2
}

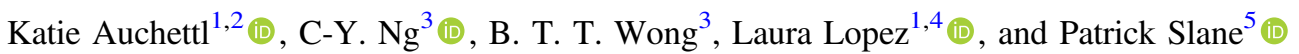 \\ ${ }^{1}$ Center for Cosmology and AstroParticle Physics (CCAPP), The Ohio State University, 191 W. Woodruff Ave., Columbus, OH 43210, USA; auchettl.1@ @osu.edu \\ ${ }^{2}$ Department of Physics, The Ohio State University, 191 W. Woodruff Ave., Columbus, OH 43210, USA \\ ${ }^{3}$ Department of Physics, The University of Hong Kong, Pokfulam Road, Hong Kong, China; ncy@bohr.physics.hku.hk \\ ${ }^{4}$ Department of Astronomy, The Ohio State University, $191 \mathrm{~W}$. Woodruff Ave., Columbus, OH 43210, USA \\ ${ }^{5}$ Harvard-Smithsonian Center for Astrophysics, 60 Garden St., Cambridge, MA 02138, USA \\ Received 2017 February 5; revised 2017 July 26; accepted 2017 July 27; published 2017 September 29
}

\begin{abstract}
We present an X-ray imaging and spectroscopic study of the molecular cloud interacting mixed-morphology supernova remnant G346.6-0.2 using XMM-Newton. The X-ray spectrum of the remnant is well described by a recombining plasma that most likely arises from adiabatic cooling and has subsolar abundances of $\mathrm{Mg}, \mathrm{Si}$, and S. Our fits also suggest the presence of either an additional power-law component with a photon index of $\sim 2$ or an additional thermal component with a temperature of $\sim 2.0 \mathrm{keV}$. We investigate the possible origin of this component and suggest that it could arise from either the Galactic ridge X-ray emission, an unidentified pulsar wind nebula, or X-ray synchrotron emission from high-energy particles accelerated at the shock. However, deeper, high-resolution observations of this object are needed to shed light on the presence and origin of this feature. Based on its morphology, its Galactic latitude, the density of the surrounding environment, and its association with a dense molecular cloud, G346.6-0.2 most likely arises from a massive progenitor that underwent core collapse.
\end{abstract}

Key words: ISM: individual (G346.6-0.2) - ISM: supernova remnants - X-rays: ISM

\section{Introduction}

Supernova remnants (SNRs) are structures that result from the explosive end of massive stars. The energy from the supernova explosion is partially converted into kinetic energy and is dissipated in collisionless shocks that heat the stellar ejecta and swept-up interstellar medium (ISM) to $\mathrm{X}$-ray-emitting temperatures. Apart from sweeping up and heating material, the shock fronts of SNRs are sites where relativistic particles can be efficiently accelerated to energies up to $10^{15} \mathrm{eV}$ (i.e., the "knee" of the cosmic-ray spectrum; Olive \& Particle Data Group 2014). Nonthermal X-ray emission arising from shock-accelerated particles has been detected in a handful of SNRs ( 14 out of 294 known Galactic SNRs). This emission is found to originate predominantly from the shell of the remnant (e.g., SN 1006: Koyama et al. 1995; RX J1713.7-3946: Koyama et al. 1997; Slane et al. 1999; and Vela Jr.: Aschenbach 1998; Slane et al. 2001) or in thin filaments at the edges of young SNRs (e.g., Tycho: Hwang et al. 2002; Warren et al. 2005; and Kepler: Cassam-Chenaï et al. 2004a), and detection of this emission provides direct evidence for electrons being accelerated to $\mathrm{TeV}$ energies. While the nonthermal $\mathrm{X}$-rays in the above remnants are confined to narrow regions close to the shock front, this does not seem to be the case for somewhat older and physically much larger SNRs like RCW 86, Vela Jr., and RX J1713.7-3946, in which the emission regions are broader and located behind the shock (Bamba et al. 2000; Slane et al. 2001; Cassam-Chenaï et al. 2004b). A number of these synchrotron X-ray-emitting SNRs such as RCW 86 and Tycho also emit noticeable thermal X-ray emission from both ejecta and shocked circumstellar material that surround the nonthermal X-ray filaments.

All nonthermal emitting SNRs detected so far are classified as shell-like, and none of these remnants are known to be interacting with nearby molecular clouds through the detection of $1720 \mathrm{MHz}$ OH maser(s) (Claussen et al. 1999). ${ }^{6}$ On the other hand, a large fraction of X-ray-emitting SNRs are known to be interacting with molecular clouds and are classified as mixedmorphology (MM) SNRs (Rho \& Petre 1998). Unlike shelltype SNRs whose X-ray emission traces a shell, MM SNRs have a centrally peaked X-ray morphology that arises from a collisionally heated plasma located in the interior of the radio shell, while often showing enhanced elemental abundances and isothermal temperatures (e.g., Lazendic \& Slane 2006).

The morphology and X-ray properties of these remnants are unexpected if one assumes standard SNR evolution models (e.g., Chevalier 1977; Truelove \& McKee 1999, 2000), and the evolutionary processes that lead to these characteristics are not well understood. There are two main models that attempt to explain the properties of MM SNRs. The first one is the thermal conduction model, in which heat and material are transported to the center of the remnant via the Coulomb collisions between electrons and ions inside the hot plasma, resulting in the centrally filled emission and isothermal temperatures (Cui \& Cox 1992; Cox et al. 1999). The second one invokes the evaporation of clumps of material that are sufficiently small and dense to not be destroyed by or disrupted by the shock itself (White \& Long 1991). Some MM SNRs also show evidence of overionization, which results from the rapid cooling of electrons and manifests itself in the form of recombination edges (e.g., Kawasaki et al. 2005; Ozawa et al. 2009; Lopez et al. 2013). This rapid cooling can occur either by adiabatic expansion (e.g., Itoh \& Masai 1989), thermal

\footnotetext{
6 Even though there are a number of methods (see Slane et al. 2015 for more details) used to infer the presence of $\mathrm{SNR} /$ molecular cloud interaction, the detection of an $\mathrm{OH}$ maser is a "smoking gun" signal, since they can only be formed in conditions related to a shock/molecular cloud interaction. A handful of these nonthermal SNRs such as RX J1713.7-3946 (Slane et al. 1999; Butt et al. 2001) show evidence of shock interaction in the form of other molecular line features, but none of these sources so far show evidence of an $\mathrm{OH}$ maser.
} 
conduction (Kawasaki et al. 2002), or the interaction with dense cavity walls or molecular clouds (Dwarkadas 2005).

Here we present an analysis of a molecular cloud interacting MM SNR G346.6-0.2, which shows evidence of a hard X-ray tail component.

G346.6-0.2 was the first to be discovered and classified as a shell-type SNR in the $480 \mathrm{MHz}$ and $5 \mathrm{GHz}$ radio bands (Clark et al. 1975) using theMolonglo Observatory Synthesis Telescope (MOST) and the Parkes $64 \mathrm{~m}$ radio telescope. The shelllike morphology of the remnant was confirmed using the Very Large Array (VLA) and MOST at $1465 \mathrm{MHz}$ and at $843 \mathrm{MHz}$, respectively (Dubner et al. 1993; Whiteoak \& Green 1996). It has an angular diameter of $8.2 \pm 0.5$ and a radio-continuum spectral index of $-0.6 \pm 0.1$ (Gaensler et al. 2001). The eastern and northwestern edges of the remnant show evidence of interaction with the surrounding environment, such as the flattening of the radio contours in these regions (Dubner et al. 1993). A number of $1720 \mathrm{MHz} \mathrm{OH}$ masers were detected along the southern rim of the radio shell at a velocity of $-76.0 \mathrm{~km} \mathrm{~s}^{-1}$ (Koralesky et al. 1998), indicating that the SNR is interacting with the surrounding molecular clouds. Koralesky et al. (1998) calculated the kinematic distance toward the masers (and thus the SNR) using the Galactic rotation curve. They determined a distance of $5.5 \mathrm{kpc}$ and $11 \mathrm{kpc}$, respectively, with a tangent point distance of $8.3 \mathrm{kpc}$ in this direction. Using the $\Sigma-D$ relationship (Huang \& Thaddeus 1985), a distance of $9 \mathrm{kpc}$ to G346.6-0.2 was suggested (Dubner et al. 1993). In this paper, we use $8.3 \mathrm{kpc}$ as the distance to the remnant, similar to that of previous studies.

In X-rays, G346.6-0.2 was first detected in the ASCA Galactic plane survey (Yamauchi et al. 2008). The ASCA GIS image shows centrally filled, diffuse $\mathrm{X}$-ray emission, indicating that this is an MM SNR. Although photon statistics were limited, Yamauchi et al. (2008) determined that the X-ray spectrum could be modeled using a thermal plasma (MEKAL model) with a temperature of $\sim 1.6 \mathrm{keV}$ or a power-law model with a photon index of $\sim 3.7$. They also derived a column density of $N_{\mathrm{H}} \sim(2-2.6) \times 10^{22} \mathrm{~cm}^{-2}$. Pannuti et al. (2014) reanalyzed the $A S C A$ data using different models (PHAB$\mathrm{S} \times$ POWERLAW, PHABS $\times$ APEC, $\mathrm{PHABS} \times \mathrm{NEI}$, and combinations of these). They found that the X-ray spectrum is best described by an absorbed nonequilibrium ionization (NEI) model with a column density of $N_{\mathrm{H}}=2.1_{-0.7}^{+0.4} \times 10^{22} \mathrm{~cm}^{-2}$, a temperature of $2.8_{-0.5}^{+1.1} \mathrm{keV}$, and an ionization timescale of $7_{-4}^{+6} \times 10^{9} \mathrm{~cm}^{-3}$ s. Based on Suzaku observations, it was claimed that the X-ray spectrum can be fitted by an absorbed hot $(k T=1.22 \pm 0.04 \mathrm{keV})$ NEI model with subsolar abundances of $\mathrm{Mg}, \mathrm{Si}, \mathrm{S}$, and $\mathrm{Fe}$, plus a power law with a photon index of $0.6 \pm 0.3$ (Sezer et al. 2011). However, a later reanalysis of the same data, after properly accounting for the strong X-ray emission from the Galactic Ridge, indicates that the X-ray emission is best described by an absorbed recombining plasma with a temperature of $0.30_{-0.01}^{+0.03} \mathrm{keV}$, subsolar abundances of $\mathrm{Mg}, \mathrm{Si}, \mathrm{S}$, and $\mathrm{Fe}$, and a column density of $N_{\mathrm{H}}=(2.3 \pm 0.1) \times 10^{22} \mathrm{~cm}^{-2}$ (Yamauchi et al. 2013).

The Spitzer IRAC survey of SNRs in the inner Galaxy (Reach et al. 2006) detected diffuse infrared emission arising from the southern rim of the radio shell of G346.6-0.2. This IR emission is coincident with the $\mathrm{OH}$ masers detected toward the south, and the IRAC colors derived in this region suggest molecular cloud interaction. There is also fainter infrared emission toward the northern edge of the remnant. The detection of spectral lines associated with shocked $\mathrm{H}_{2}$ emission from G346.6-0.2 using Spitzer IRS observations (Hewitt et al. 2009) indicates that the remnant is interacting with a highdensity environment such as a molecular cloud. However, no $\gamma$-ray emission was found, using approximately 3.5 years of PASS 7 Fermi-LAT data (Ergin \& Ercan 2012).

In this paper, we present an X-ray observation of SNR G346.6-0.2 using XMM-Newton. In Section 2, we describe the $X M M$ data reduction, the spatial and spectral analysis, and our point source analysis. In Sections 3 and 4 we infer the properties of G346.6-0.2, and we discuss the origin and nature of its thermal and nonthermal X-ray emission, while in Section 5 we discuss the possible origin of the nonthermal component. In Section 6 we discuss the nature of the point sources we detected and search for a potential neutron star candidate, while in Section 7 we summarize our results.

\section{XMM-Newton Observations, Analysis, and Results}

SNR G346.6-0.2 was observed with both the MOS and PN detectors on board the XMM-Newton Observatory on 2011 March 11 for a total of $30.1 \mathrm{ks}$ (ObsID:0654140101). The telescope was pointed at $(\alpha, \delta)=\left(17^{\mathrm{h}} 09^{\mathrm{m}} 59^{\mathrm{s}} .8,-40^{\circ} 12^{\prime} 56^{\prime \prime} .4\right)$, and the MOS and PN detectors were operated in the full-frame mode with the thick filter, with the SNR fully enclosed by the field of view of the detectors. We performed the data reduction and analysis using the XMM-Newton science system (SAS) version 14.0.0 with CALDB 4.6.7. ${ }^{8}$

Before completing our imaging and spectral analysis, we first checked for periods of high background or proton flares by generating a count rate histogram using events with energy between 10 and $12 \mathrm{keV}$ for the observation. We find that our observation is only slightly affected by high background or flares, giving effective exposures of $29.4 \mathrm{ks}, 29.3 \mathrm{ks}$, and $24.6 \mathrm{ks}$ for MOS1, MOS2, and PN, respectively. As suggested in the SAS analysis threads ${ }^{9}$ and XMM-Newton Users Handbook, ${ }^{10}$ we reduced the data following the standard screening of events, with single to quadruple events (PATTERN $\leqslant 12$ ) chosen for the MOS detectors, while for the PN detector only single and double events (PATTERN $\leqslant 4$ ) were selected. We also used the standard canned screening set of FLAGS for both the MOS (\#XMMEA_EM) and PN (\#XMMEA_EP) detectors.

As G346.6-0.2 is located along the Galactic plane, both Galactic Ridge X-ray emission (GRXE) and the cosmic X-ray background (CXB) can contribute nonnegligibly to the observed emission. To correct for this, we must take vignetting effects into account. Thus, we process all event files using the task evigweight, which weights each event by an energydependent factor that is equivalent to the ratio of the effective area at the center of the observation and the effective area at the position of interest. ${ }^{11}$ All analysis products and results presented below are extracted from these cleaned, filtered, and vignetting-corrected event files.

\footnotetext{
7 https://www.cosmos.esa.int/web/xmm-newton/documentation/

8 https://www.cosmos.esa.int/web/xmm-newton/calibration

9 https://www.cosmos.esa.int/web/xmm-newton/sas-threads

10 https://xmm-tools.cosmos.esa.int/external/xmm_user_support/ documentation/sas_usg/USG/

11 See https://xmm-tools.cosmos.esa.int/external/sas/current/doc/ evigweight for more details.
} 

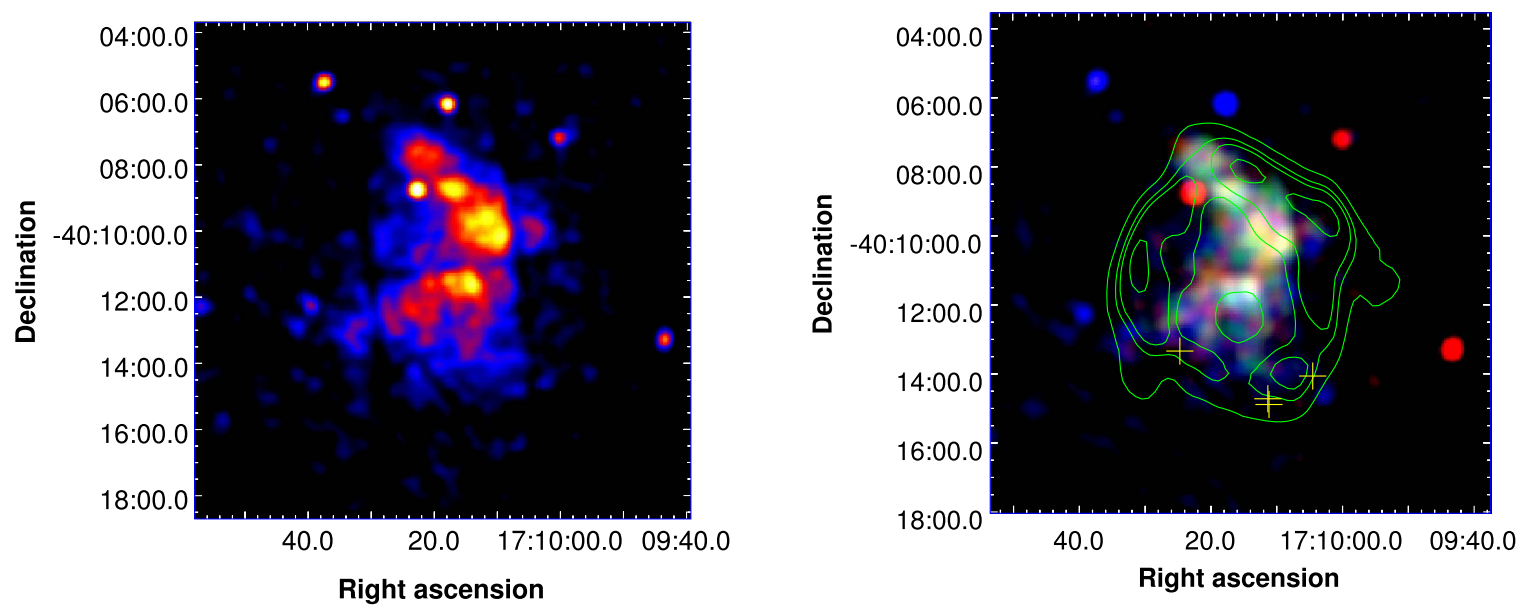

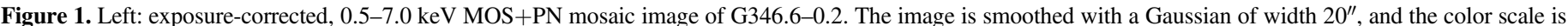

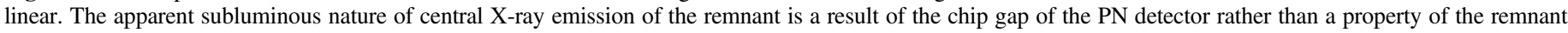

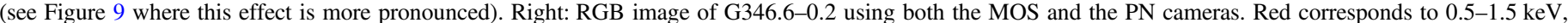

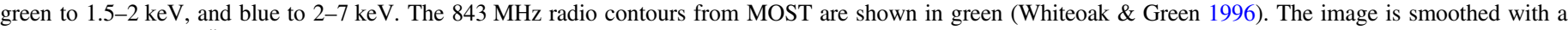
Gaussian of width $20^{\prime \prime}$. The yellow crosses indicate the locations of the $1720 \mathrm{MHz}$ OH masers detected with the VLA (Koralesky et al. 1998).

\subsection{Imaging Analysis}

We used the SAS task emosaic to combine the MOS and PN observations to produce a single exposure-corrected intensity image of the entire SNR. The resulting image in the $0.5-7 \mathrm{keV}$ energy band is shown in Figure 1 (left). To determine any possible spectral variations of the remnant and the nature of point sources in the field, we also generated an RGB image using events from both the MOS and PN detectors (Figure 1 right).

These images reveal that the X-ray emission is relatively clumpy in nature. The bulk of the X-ray emission found toward the north of the remnant produces a significant amount of soft $\mathrm{X}$-rays, while the emission seen toward the southern region overlapping the positions of the $\mathrm{OH}$ masers is harder in nature. The X-ray emission from the SNR shows an arc-like morphology, and it is brightest toward the west. The emission extends to both the northeast and southeast, with the northern extension seeming to follow the slight protrusion of the radio contours, as seen in the RGB image (Figure 1, right). The $\mathrm{X}$-ray emission of this remnant is surrounded by faint diffuse emission and is fully enclosed by the MOST radio contours (Whiteoak \& Green 1996), covering a region with an approximate size of $7 ! 3 \times 8 ! 2$.

There are a few bright point sources immediately surrounding the SNR (see Figure 1). One of them coincides with the radio shell in the north of the remnant, and another is found in the southwest near the $\mathrm{OH}$ masers. All other point sources are found outside the radio remnant shell. The point sources surrounding the remnant emit strongly in either soft or hard $\mathrm{X}$-rays only. In particular, the point source within the northern part of the radio shell is dominated by soft X-rays. A more detailed analysis and discussion of the point sources in the field of view are found in Section 2.3.

We also note that there is an arc-like feature toward the southeast of the remnant, which could arise from either a bright nearby source or from flaring (see Figure 2). As we filtered out periods of high background or proton flares before analyzing the data products of this observation, it is more likely that these fringes arise from a nearby source. We searched the ROSAT All-Sky Survey Bright Source Catalog (Voges et al. 1999) and the Third XMM-Newton Serendipitous Source Catalog (Rosen et al. 2016) and find the low-mass X-ray binary 4U 1708-40 (1RXS J171224.8-405034) located approximately 0.8 from G346.6-0.2, making it likely to be the source of this emission.

\subsection{Spectral Analysis}

To determine the spectral properties of G346.6-0.2, we extract spectra from six regions shown in Figure 3 (right) using the SAS task evselect and the cleaned, vignetting-corrected event files from all three EPIC cameras. In addition, we extracted a global spectrum from an elliptical region centered on $(\alpha, \delta)=\left(17^{\mathrm{h}} 10^{\mathrm{m}} 17^{\mathrm{s}} .6,-40^{\circ} 10^{\prime} 29^{\prime \prime} .3\right)$ with semiminor and semimajor radii of $3^{\prime}$ and $3 ! 4$, respectively, which encloses the bulk of the SNR X-ray emission. Point sources overlapping these regions were excluded. For each region, we extracted spectral response and effective area files using the tasks arfgen and rmfgen. To account for the background, we selected an annulus region that directly surrounds the emission of the remnant (see Figure 3 left). We excluded both point sources and the arc-shaped fringes from singly reflected X-rays seen in all observations from our background region.

The spectral fitting was performed using the X-ray analysis software XSPEC version 12.9.0c, over an energy range of 0.7-7 keV. We also used AtomDB 3.0.2 ${ }^{12}$ (Smith et al. 2001; Foster et al. 2012). Each spectrum was grouped with a minimum of 20 counts per energy bin and fitted using $\chi^{2}$ statistics. To investigate the emission of the remnant, we used an NEI collisional plasma model, VRNEI, ${ }^{13}$ which is characterized by a final $(k T)$ and initial electron temperature $\left(k T_{\text {init }}\right)$, elemental abundances, and a single ionization timescale $\left(\tau=n_{e} t\right)$. This model allows one to simulate the thermal emission from either a plasma that is ionizing up to or is in ionization equilibrium (i.e., $k T_{\text {init }}<k T$ ), or a recombining plasma that was in collisional equilibrium with $k T_{\text {init }}$ and then suddenly cooled to its final temperature $k T$ (i.e., $k T_{\text {init }}>k T$ ). We also used an absorbed APEC model (Smith et al. 2001), which allows one to model a plasma that is in collisional ionization equilibrium (CIE).

\footnotetext{
12 http://www.atomdb.org/

13 https://heasarc.gsfc.nasa.gov/xanadu/xspec/manual/node210.html
} 


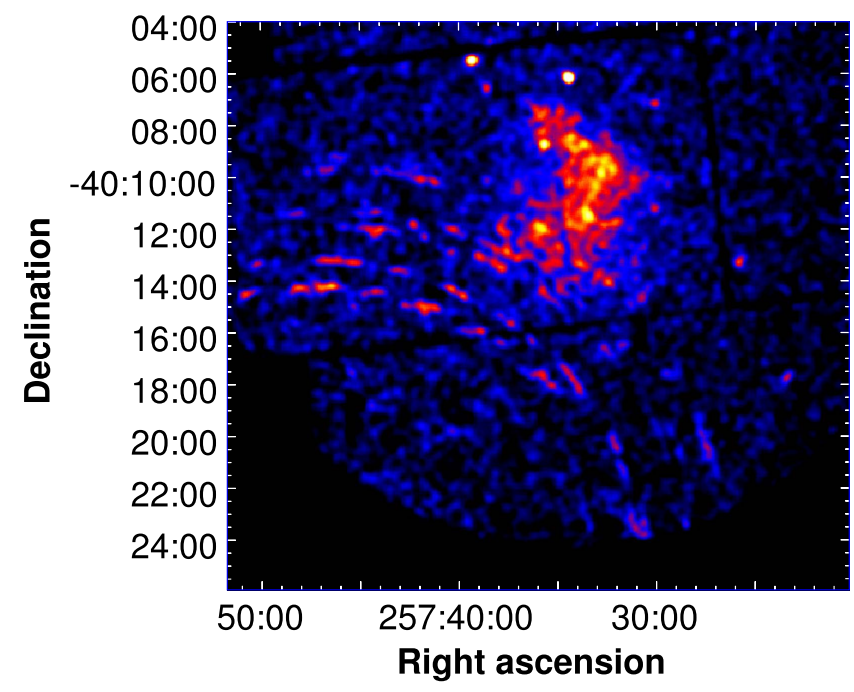

Figure 2. MOS2 image of G346.6-0.2 showing the singly reflected X-rays from a nearby source in the form of large arcs toward the southeast of the remnant. This artifact is seen in all detectors and most prominently in MOS2.

The foreground absorption column density $N_{\mathrm{H}}$ was modeled using TBABS (Wilms et al. 2000). Due to the presence of noticeable emission lines from $\mathrm{Mg}, \mathrm{Si}$, and $\mathrm{S}$, the abundances of these elements were also let free in the VRNEI model fit. All other elemental abundances were fixed at the solar values (Wilms et al. 2000). We find that all regions favored $k T_{\text {init }}>k T$, implying that the X-ray emission from G346.6-0.2 arises from a recombining plasma, similar to what was suggested previously (Yamauchi et al. 2013). For completeness, we also attempted to fit the emission of the remnant using a TBABS $\times$ VRNEI model with $k T_{\text {init }}<k T$ or a TBABS $\times$ APEC model. However, we find that both these models produced a worse fit (reduced $\chi^{2} \sim 1.3$ for both models), and they suggest extremely high temperatures for the SNR (i.e., $k T_{e} \sim 1.7-2.6 \mathrm{keV}$ ). In Table 1 , we list the best-fit parameters and their $90 \%$ confidence level uncertainties, while in Figure 4 we plot the global X-ray spectrum of G346.6-0.2.

We attempt to fit the value of $k T_{\text {init }}$, but we found that it is poorly constrained for all individual regions except for the global spectrum, for which we obtained $k T_{\text {init }}=6_{-1}^{+4} \mathrm{keV}$. Therefore, we fixed $k T_{\text {init }}$ at the global value when fitting the spectra from individual regions.

Overall, a TBABS $\times$ VRNEI model with underabundant $\mathrm{Mg}$, $\mathrm{Si}, \mathrm{S}$, and $\mathrm{Fe}$, a plasma temperature of $k T \sim 0.30 \mathrm{keV}$, and $k T_{\text {init }}$ fixed at $6 \mathrm{keV}$ was able to fit the global spectrum reasonably well with a reduced $\chi^{2}=1.16$. This is consistent with that derived by Yamauchi et al. (2013) using Suzaku data. However, this model fails to fit the high-energy spectrum above $\sim 3 \mathrm{keV}$, as shown by the residuals in Figure 4 (left panel). The Suzaku X-ray spectrum and model fits presented in Table 1 of Yamauchi et al. (2013) also hint at an additional spectral component. ${ }^{14}$ To account for this excess seen in the hard X-ray band (Figure 4 left panel), we attempted to add a recombining plasma (RNEI) or an NEI model component, but we found that these models did not improve the fit when $\tau<10^{12} \mathrm{~cm}^{-3} \mathrm{~s}$. However, we find that these models significantly improve the

\footnotetext{
${ }^{14}$ The best-fit recombining NEIJ model presented by Yamauchi et al. (2013) has a reduced $\chi^{2}$ between 1.20 and 1.40 depending on the background they used, but the poor statistics above $\sim 4 \mathrm{keV}$ precludes a detailed study of this component in their paper.
}

fit when $\tau>10^{13} \mathrm{~cm}^{-3} \mathrm{~s}$, mimicking that of an APEC model, which we discuss further below.

It is possible that this hard X-ray tail could arise from either excess thermal emission from the Galactic Ridge or from a power-law component that arises from a nonthermal population of the electrons accelerated by the supernova shock or from an unseen pulsar wind nebula (PWN). To test these possibilities, we added either an additional APEC model (Smith et al. 2001) or a power-law model in which we let both the temperature and the photon index be free, respectively.

We find that adding a power-law component significantly improves the fit and gives a reduced $\chi^{2}$ of 0.99 , and an $F$ test indicates a null hypothesis probability of $1.0 \times 10^{-15}$ when compared with our best-fit TBABS $\times$ VRNEI model. The same is also true for individual spectra except region 2 ; all have an $F$ test null hypothesis probability of $<10^{-5}$. The power-law component for the global spectrum has a photon index of $\Gamma=2.0_{-0.9}^{+0.7}$, while the individual regions have $\Gamma$ between 1.0 and 2.5 (see Table 1). The power-law index implied by our fits is similar to that obtained by Yamauchi et al. (2008), whose power-law model fit of ASCA observations of the remnant required a power-law index $>1.7$. These values are much larger than $\Gamma \sim 0.5$ obtained with Suzaku using a WABS*(VNEI +POWERLAW) model (Sezer et al. 2011), but these authors did not correct for vignetting. In Table 1 we have listed the results of our fits using either a TBABS $\times$ VRNEI or TBABS $\times($ VRNEI+POWERLAW $)$ model.

Similar to our power-law model, we find that an additional APEC model also improves the fit to our global and individual spectra (reduced $\chi^{2}$ of 0.96 for the global spectrum). Here the best-fit APEC temperature derived from the global spectrum is $1.9_{-0.3}^{+0.6} \mathrm{keV}$, while those for the individual regions are similar, albeit with larger uncertainties (see Table 2). This temperature is much higher than that expected from an SNR, or from the $0.79 \mathrm{keV}$ excess emission seen directly surrounding G346.6-0.2 (Yamauchi et al. 2013), while it is lower than that derived by Pannuti et al. (2014) using a single APEC model.

The derived temperature value is higher than that estimated by various studies of the Galactic Ridge emission using X-rays (e.g., Yuasa et al. 2012; Uchiyama et al. 2013; Yamauchi et al. 2014; Nobukawa et al. 2016); however, within uncertainties, it is consistent with that of the low-temperature component of the Galactic Ridge emission derived by, for example, Yuasa et al. (2012) and Uchiyama et al. (2013). We find that the properties of the VRNEI models in these fits are comparable to those in the VRNEI+POWERLAW fits (see Table 1).

We find that both the global and individual regions have an ionization timescale of $\sim 4.8 \times 10^{11} \mathrm{~cm}^{-3} \mathrm{~s}$, indicating that the plasma is far from ionization equilibrium. This is similar to the values derived by Yamauchi et al. (2013) and Sezer et al. (2011), but is significantly different from the ASCA and Suzaku results (Yamauchi et al. 2008; Pannuti et al. 2014). The average temperature of the recombining plasma is $0.26 \mathrm{keV}$, which is slightly lower than the $0.30_{-0.01}^{+0.03} \mathrm{keV}$ reported by Yamauchi et al. (2013) and lower than the temperature of $0.30 \mathrm{keV}$ derived using only a TBABS $\times$ VRNEI model.

As Yamauchi et al. (2008), Sezer et al. (2011), and Pannuti et al. (2014) used a CIE model or an NEI model without overionization to describe the recombining plasma, our results differ from their studies. In addition, Yamauchi et al. (2008) and Sezer et al. (2011) did not properly account for either the 

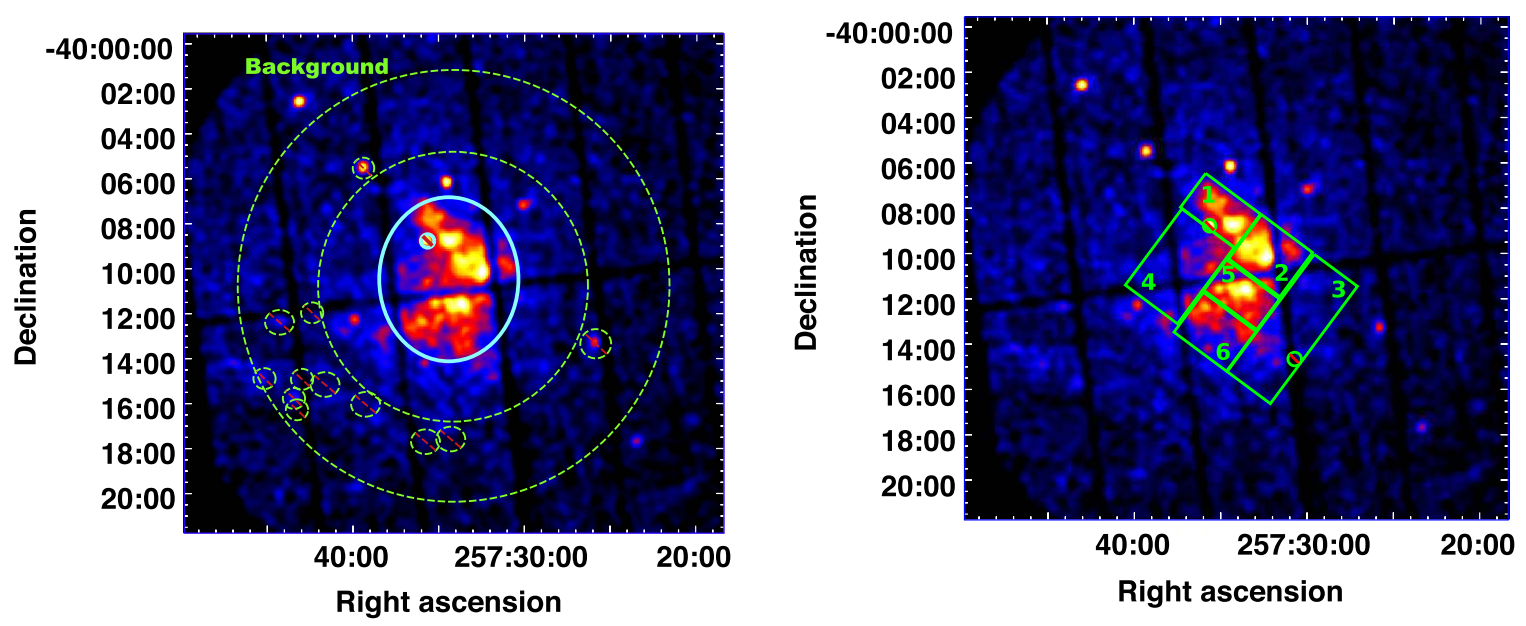

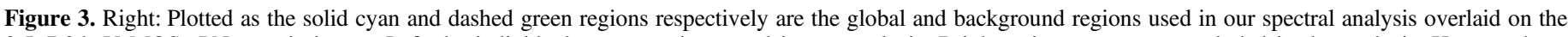

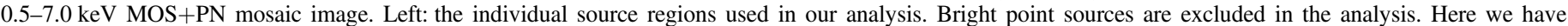
adjusted the contrast of the image such that only the brightest emission from the remnant is observed.

non-X-ray background of Suzaku or vignetting effects, which can lead to the plasma temperature being much higher in their model fits to compensate for the excess flux above $\sim 5 \mathrm{keV}$ (Yamauchi et al. 2013). Another difference is that we use updated elemental abundances (Wilms et al. 2000) and atomic cross sections (ATOMDB 3.0.2) in the analysis, compared to much older versions (e.g., Anders \& Grevesse 1989 abundances or ATOMDB 2.0) used in previous studies.

The global and individual spectra, except for region 3, all suggest underabundance of $\mathrm{Mg}$. In addition, the global and regions 1, 2, and 4 spectra require underabundance of $\mathrm{Si}$. Underabundance of $\mathrm{S}$ is required for the global and regions 1, 2, 4, and 5 spectra. This is similar to the Suzaku results (Sezer et al. 2011; Yamauchi et al. 2013). However, we do not find evidence for underabundance of $\mathrm{Fe}$ or overabundance of $\mathrm{Ca}$ as claimed by the above two studies. Note that the ASCA data were unable to verify these, due to limited photon statistics (Yamauchi et al. 2008; Pannuti et al. 2014).

We derived a hydrogen column density of $N_{\mathrm{H}}=$ $(2.0-3.3) \times 10^{22} \mathrm{~cm}^{-2}$. It is highest in regions 1 and 4 in the eastern side of the remnant and lowest on the western side (region 3). The $N_{\mathrm{H}}$ values are comparable to those previously reported (Yamauchi et al. 2008; Sezer et al. 2011; Yamauchi et al. 2013; Pannuti et al. 2014).

Finally, to check the possibility that the additional component could arise from the singly reflected X-rays seen in Figure 2, we estimate the flux contribution of this artifact using WebPIMMS. ${ }^{15}$ Assuming the $N_{\mathrm{H}}$ and $\Gamma$ derived from our global X-ray spectrum, this corresponds to an absorbed flux of $\sim 1 \times 10^{-14} \mathrm{erg} \mathrm{cm}^{-2} \mathrm{~s}^{-1}$ over the $0.5-7.0 \mathrm{keV}$ energy band. This is only $\sim 2 \%$ of the absorbed flux of the power-law component in both the global spectrum and in all regions that require an additional power law.

\subsection{Point Source Analysis}

We identified X-ray point sources in the field of view by running the task edetect_chain on the PN data in five standard energy bands $(0.2-0.5 \mathrm{keV}, 0.5-1 \mathrm{keV}, 1-2 \mathrm{keV}$, $2-4.5 \mathrm{keV}$, and $4.5-10 \mathrm{keV})$. We chose a likelihood threshold value of $30 \sigma$ to mitigate background contamination. A total of 25

\footnotetext{
15 https://heasarc.gsfc.nasa.gov/cgi-bin/Tools/w3pimms/w3pimms.pl
}

bright point sources were detected in the field. Their positions and count rates in the soft $(0.2-2 \mathrm{keV})$ and hard $(2-10 \mathrm{keV})$ bands are listed in Tables 3 and 4 . We calculated the hardness ratio (HR) using $\left(R_{2-10}-R_{0.2-2}\right) /\left(R_{2-10}+R_{0.2-2}\right)$, where $R$ are the count rates in the soft and hard bands from the PN detector. We use only the events from the PN detector for this analysis because of its high sensitivity and large effective area. Also, a few sources fall off the MOS detectors or are located on CCD6 of MOS1, which is no longer operational.

For sources from which we were able to extract sufficient counts and thus extract an X-ray spectrum, we grouped the spectra with a minimum of 10 counts per bin and modeled each spectrum using an absorbed power law. We compared the positions of the X-ray sources with the UNSO-B1 (Monet et al. 2003) and 2MASS (Skrutskie et al. 2006) catalogs to identify any optical or infrared counterparts, respectively. We considered only optical and infrared sources within $3 \sigma$ uncertainties of the X-ray positions, and we identified 14 optical counterparts. No optical counterparts are found within $3 \sigma$ uncertainties for the other 11 sources. The results of our point source analysis are listed in Tables 3 and 4.

\section{Possible Origin of the Center-filled X-Ray Emission}

The detection of $\mathrm{OH}$ masers coincident with G346.6-0.2 implies that this remnant is expanding into a dense and nonuniform environment. The characteristics of MM SNRs are thought to arise from the interaction between the SNR and this dense molecular material. However, their morphological and plasma properties are difficult to explain using the standard Sedov SNR evolution model (see, e.g., Long et al. 1991; Safi-Harb et al. 2000). Two popular models ${ }^{16}$ used to explain the properties of MM SNRs are thermal conduction (e.g., Cui \& Cox 1992; Cox et al. 1999) and the evaporation of dense clumps of material inside the remnant (e.g., White \& Long 1991).

\footnotetext{
${ }^{16}$ There are other models that attempt to explain the properties of MM SNRs. Petruk (2001) suggested that the central X-ray emission of MM SNRs arises from projection effects, while Dwarkadas (2005) suggested that their properties arise from the collision of the SNR blast wave with the dense cavity walls or ring-like structures that are produced by massive progenitors as they lose mass. However, a detailed comparison between models is beyond the scope of this paper.
} 
Table 1

Best-fit Parameters for the Global Spectrum and Individual Regions Using an Absorbed VRNEI+POWERLAW Model

\begin{tabular}{|c|c|c|c|c|c|c|c|c|c|c|c|}
\hline Region & $\left(10^{22_{\mathrm{H}} \mathrm{cm}^{-2}}\right)$ & $\begin{array}{c}k T \\
(\mathrm{keV})\end{array}$ & $\begin{array}{l}k T_{\text {init }} \\
(\mathrm{keV})\end{array}$ & $\mathrm{Mg}$ & $\mathrm{Si}$ & S & $\begin{array}{c}\tau=n_{e} t \\
\left(10^{11} \mathrm{~cm}^{-3} \mathrm{~s}\right)\end{array}$ & $F_{\text {vrnei }}{ }^{\mathrm{b}, \mathrm{c}}$ & $\Gamma$ & $F_{\mathrm{pwl}}^{\mathrm{b}, \mathrm{c}}$ & $\chi_{\nu}^{2}$ (dof) \\
\hline Global & $3.1_{-0.2}^{+0.1}$ & $0.26 \pm 0.02$ & $6_{-1}^{+4}$ & $0.38 \pm 0.1$ & $0.73 \pm 0.1$ & $0.60 \pm 0.01$ & $5.4 \pm 0.7$ & $11 \pm 2$ & $2.0_{-0.9}^{+0.7}$ & $3.3 \pm 2$ & $0.99(922)$ \\
\hline 1 & $3.3 \pm 0.2$ & $0.23 \pm 0.02$ & $6^{\mathrm{a}}$ & $0.12 \pm 0.1$ & $0.46 \pm 0.07$ & $0.38_{-0.07}^{+0.09}$ & $5.3_{-0.6}^{+0.8}$ & $5.4_{-1.0}^{+0.5}$ & $1.8_{-1.1}^{+1.0}$ & $1.6_{-2}^{+5}$ & $1.04(651)$ \\
\hline 2 & $2.9 \pm 0.2$ & $0.24 \pm 0.02$ & $6^{\mathrm{a}}$ & $0.17 \pm 0.1$ & $0.63_{-0.08}^{+0.09}$ & $0.50_{-0.07}^{+0.08}$ & $3.8 \pm 0.5$ & $9.4 \pm 0.03$ & $\ldots$ & $\ldots$ & $0.99(710)$ \\
\hline 3 & $2.0 \pm 0.3$ & $0.21 \pm 0.04$ & $6^{\mathrm{a}}$ & & $\ldots$ & & $4.0_{-1.1}^{+0.8}$ & $5.3 \pm 2$ & $1.0_{-0.8}^{+1.1}$ & $3.7_{-5}^{+6}$ & $1.01(836)$ \\
\hline 4 & $3.4 \pm 0.2$ & $0.26 \pm 0.02$ & $6^{\mathrm{a}}$ & $0.23 \pm 0.14$ & $0.56_{-0.08}^{+0.09}$ & $0.51_{-0.09}^{+0.07}$ & $5.3_{-0.6}^{+0.7}$ & $12_{-5}^{+2}$ & $1.5_{-1.2}^{+0.9}$ & $4.0_{-2}^{+7}$ & 1.03 \\
\hline 5 & $2.8_{-0.3}^{+0.2}$ & $0.24 \pm 0.04$ & $6^{\mathrm{a}}$ & $0.29 \pm 0.2$ & $\ldots$ & $0.54_{-0.1}^{+0.2}$ & $4.3_{-0.4}^{+0.9}$ & $5.7_{-1}^{+2}$ & $2.5_{-1.2}^{+0.4}$ & $3.5_{-2}^{+3}$ & 1.04 \\
\hline 6 & $2.9_{-0.3}^{+0.2}$ & $0.28 \pm 0.03$ & $6^{\mathrm{a}}$ & $0.42 \pm 0.2$ & $\ldots$ & .. & $5.4 \pm 0.8$ & $5.8_{-1}^{+2}$ & $2.1_{-0.8}^{+0.4}$ & $4.1 \pm 3$ & $1.03(621)$ \\
\hline
\end{tabular}

Notes. All uncertainties correspond to the $90 \%$ confidence level.

${ }^{\mathrm{a}}$ We fix $k T_{\text {init }}$ for all individual regions at the global best-fit value.

${ }^{b}$ Absorbed X-ray fluxes in the $0.5-7 \mathrm{keV}$ energy range.

${ }^{\mathrm{c}}$ Flux units: $10^{-13} \mathrm{erg} \mathrm{cm}^{-2} \mathrm{~s}^{-1}$. 

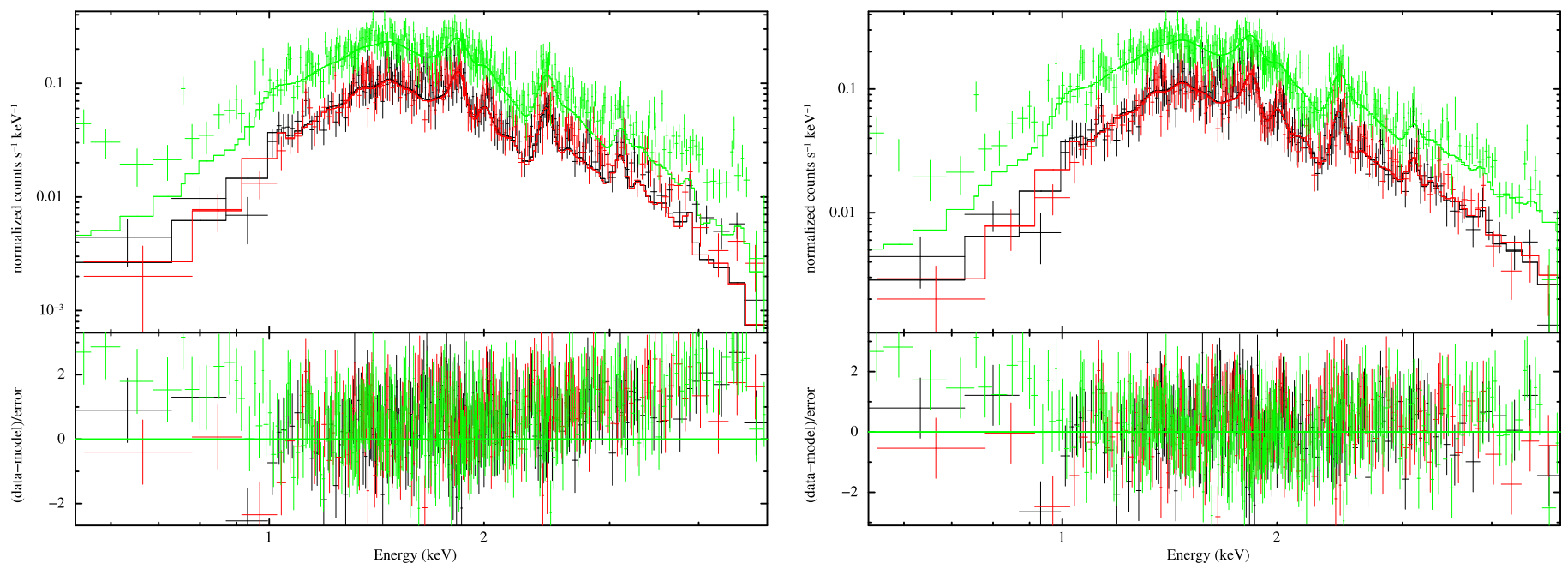

Figure 4. Left: best-fit absorbed VRNEI model for the MOS1 (black), MOS2 (red), and PN (green) spectra, with a temperature of $0.34 \pm 0.01 \mathrm{keV}, k T_{\text {init }}$ fixed at $6 \mathrm{keV}$, and underabundant $\mathrm{Mg}, \mathrm{Si}, \mathrm{S}$, and Fe. Right: best-fit absorbed VRNEI+POWERLAW model with a temperature of $0.20_{+0.02}^{+0.03} \mathrm{keV}, k T_{\text {init }}=6 \mathrm{keV}$, underabundant $\mathrm{Mg}, \mathrm{Si}$, and $\mathrm{S}$, and a power-law index of $\Gamma=2.3_{-0.8}^{+0.5}$. The left panel shows that the single absorbed VRNEI model significantly underestimates the flux above $\sim 4 \mathrm{keV}$.

In the thermal conduction model (Cui \& Cox 1992; Cox et al. 1999), the material immediately behind the shock front begins to cool after the passage of the supernova blast wave. If thermal conduction is the main source of cooling, this results in the transport of heat and material to the center of the remnant, increasing its central density and smoothing the temperature gradient behind the shock. At the shock front itself, the relatively high density of the swept-up ISM absorbs X-ray emission from the shell, while only the central emission is observable. This model is able to reproduce the center bright X-ray emission of a sample of SNRs such as W44 (e.g., Cox et al. 1999); however, it is unable to fully explain the temperature and brightness distributions of other MM SNRs.

In the White \& Long (1991) model, the properties of MM SNRs arise from the evaporation of dense clumps of material. The SNR is assumed to be evolving in a medium that contains many cold cloudlets that are sufficiently small and dense that they do not affect the passage of the shock and are neither destroyed nor swept up. Once the shock has passed, the cloudlets are then embedded in the hot postshock plasma and evaporate via thermal conduction. This fills the SNR interior with a relatively dense gas that emits X-rays.

Even though neither of these two models are able to reproduce completely the complex emission, morphology, and dynamical properties of all MM SNRs, they provide us with a good handle on their properties (e.g., Slane et al. 2002; Gelfand et al. 2013). As G346.4-0.2 is expanding in a highly inhomogeneous medium rather than an environment with a strong density gradient, we adopt the White \& Long (1991) model to investigate the properties of this remnant.

\subsection{Evaporation of Dense Clumps}

When the mass of the swept-up ISM is greater than the ejecta mass, the SNR enters the Sedov-Taylor phase (Taylor 1950; Sedov 1959). At this point, the ejecta have been shocked and can be approximated assuming that it is adiabatically expanding in a uniform medium. White \& Long (1991) generalized the Sedov model and its corresponding similarity solution such that it could describe the evolution of SNRs in a dense, nonuniform environment, which takes into account the evaporation of dense clumps of material. White \& Long (1991) introduce two additional parameters to the Sedov model: $\mathcal{C}$, the ratio of the ISM cloud mass to that of the intercloud medium, and $\tau$, the ratio of the cloud evaporation timescale to the SNR age. For appropriate values of $\mathcal{C}$ and $\tau$, one is able to reproduce the bright X-ray morphologies of MM SNRs while also being able to recover the standard Sedov (1959) model when $\mathcal{C}, \tau \ll 1$ or $\tau \gg \mathcal{C}$.

To characterize the properties of G346.6-0.2, we sample a wide range of values for the shock temperature $\left(k T_{s}\right), \mathcal{C}$, and $\tau$ to determine the White \& Long (1991) model that best reproduces the properties of the remnant. For each set of parameters, we derived the model surface brightness and $\mathrm{X}$-ray temperature profiles assuming the $\mathrm{X}$-ray emissivities of a plasma with an X-ray temperature $\left(k T_{X}\right)$, ionization timescale, and abundances similar to what we obtained from our spectral analysis of the global X-ray spectrum of the remnant. The X-ray emissivity was calculated assuming the observed column density and using the spectral response files of the MOS1 detector. For each set of parameters, we calculate the average model $\mathrm{X}$-ray temperature from the corresponding temperature profiles, and we compare this result to the $\mathrm{X}$-ray temperature derived from modeling the global X-ray spectrum of the remnant.

We derived the surface brightness profile using the MOS1 observation, as the SNR overlaps with a large number of chip gaps in the PN data. In addition, we used an annulus centered at $(\alpha, \delta)=\left(17^{\mathrm{h}} 10^{\mathrm{m}} 17^{\mathrm{s}},-40^{\circ} 10^{\prime} 59^{\prime \prime}\right)$ that fully encloses the $\mathrm{X}$-ray emission of the remnant. We also exclude all bright point sources that we found using our point source analysis discussed in Section 2.3.

To estimate the values of $k T_{s}, \mathcal{C}$, and $\tau$ favored by the global $\mathrm{X}$-ray temperature and the surface brightness profile of G346.4 -0.2 , we randomly sampled the parameter space of each variable. To determine the best-fit values of these parameters, we derived a likelihood function for each variable. A likelihood function, $\mathcal{L}$, is the probability of obtaining the observed data $d_{i}$ 
Table 2

Best-fit Parameters for the Global Spectrum and Individual Regions Using an Absorbed VRNEI+APEC Model

\begin{tabular}{|c|c|c|c|c|c|c|c|c|c|c|c|}
\hline Region & $\left(10^{22} \mathrm{~cm}_{\mathrm{H}}^{N_{\mathrm{H}}}\right)$ & $\begin{array}{c}k T \\
(\mathrm{keV})\end{array}$ & $\begin{array}{l}k T_{\text {init }} \\
(\mathrm{keV})\end{array}$ & $\mathrm{Mg}$ & $\mathrm{Si}$ & S & $\begin{array}{c}\tau=n_{e} t \\
\left(10^{11} \mathrm{~cm}^{-3} \mathrm{~s}\right)\end{array}$ & $F_{\text {vrnei }}{ }^{\mathrm{b}, \mathrm{c}}$ & $k T_{\text {apec }}$ & $F_{\text {apec }}^{\mathrm{b}, \mathrm{c}}$ & $\chi_{\nu}^{2}(\mathrm{dof})$ \\
\hline$\overline{\text { Global }}$ & $3.0 \pm 0.2$ & $0.24 \pm 0.02$ & $6_{-2}^{+9}$ & $0.34 \pm 0.1$ & $0.68 \pm 0.1$ & $0.49 \pm 0.1$ & $5.3 \pm 0.6$ & $8.8 \pm 0.05$ & $1.9_{-0.3}^{+0.6}$ & $5.2 \pm 0.3$ & $0.96(899)$ \\
\hline 1 & $3.3 \pm 0.2$ & $0.23 \pm 0.02$ & $6^{\mathrm{a}}$ & $0.08_{-0.04}^{+0.10}$ & $0.45 \pm 0.1$ & $0.36 \pm 0.1$ & $5.8 \pm 1$ & $5.0_{-0.6}^{+0.2}$ & $2.4 \pm 0.4$ & $2.3 \pm 0.2$ & $1.06(651)$ \\
\hline 2 & $2.9 \pm 0.2$ & $0.24 \pm 0.02$ & $6^{\mathrm{a}}$ & $0.17 \pm 0.1$ & $0.63_{-0.08}^{+0.09}$ & $0.50_{-0.07}^{+0.08}$ & $3.8 \pm 0.5$ & $9.4 \pm 0.03$ & $\ldots$ & $\ldots$ & $0.99(710)$ \\
\hline 3 & $2.00 \pm 0.2$ & $0.21 \pm 0.03$ & $6^{\mathrm{a}}$ & $\cdots$ & $\cdots$ & $\ldots$ & $4.0_{-1.1}^{+0.8}$ & $4.5_{-0.2}^{+0.8}$ & $2.5_{-0.7}^{+10}$ & $3.6 \pm 0.3$ & $1.03(826)$ \\
\hline 4 & $3.5 \pm 0.2$ & $0.22 \pm 0.2$ & $6^{\mathrm{a}}$ & $0.22 \pm 0.2$ & $0.54 \pm 0.1$ & $0.50 \pm 0.1$ & $6.0_{-0.6}^{+0.7}$ & $8.8_{-0.5}^{+0.3}$ & $1.7 \pm 0.2$ & $5.2 \pm 0.2$ & $1.02(969)$ \\
\hline 5 & $2.7 \pm 0.2$ & $0.24 \pm 0.03$ & $6^{\mathrm{a}}$ & $0.28 \pm 0.2$ & $\cdots$ & $0.57 \pm 0.1$ & $4.3 \pm 0.6$ & $5.6_{-0.7}^{+0.3}$ & $2.3_{+6.0}^{-0.5}$ & $2.8 \pm 0.1$ & $1.04(585)$ \\
\hline 6 & $2.9_{-0.3}^{+0.2}$ & $0.28 \pm 0.03$ & $6^{\mathrm{a}}$ & $0.43 \pm 0.2$ & $\ldots$ & $\ldots$ & $5.8 \pm 0.9$ & $5.3_{+1.4}^{-0.8}$ & $3.4_{-1.1}^{+6.0}$ & $3.8 \pm 0.1$ & $1.03(621)$ \\
\hline
\end{tabular}

Notes. All uncertainties correspond to the $90 \%$ confidence level.

${ }^{\mathrm{a}}$ We fix $k T_{\text {init }}$ for all individual regions at the global best-fit value.

${ }^{b}$ Absorbed X-ray fluxes in the $0.5-7 \mathrm{keV}$ energy range.

${ }^{\mathrm{c}}$ Flux units: $10^{-13} \mathrm{erg} \mathrm{cm}^{-2} \mathrm{~s}^{-1}$. 
Table 3

Position of the Optical/UV/X-Ray Counterparts Found within $3 \sigma$ of the X-Ray Sources in the Field of View

\begin{tabular}{|c|c|c|c|c|c|c|}
\hline $\mathrm{Src}$ & R.A. & Decl. & $\begin{array}{l}\text { Positional } \\
\text { Uncert. (") }\end{array}$ & UNSO-B $1^{\mathrm{a}}$ & 2 MASS $^{\mathrm{a}}$ & $3 \mathrm{XMM}$ \\
\hline 1 & $17: 10: 52.32$ & $-40: 02: 35.52$ & 0.5 & $\ldots$ & $\ldots$ & $\ldots$ \\
\hline 2 & $17: 10: 37.44$ & $-40: 05: 31.56$ & 0.5 & $\ldots$ & $\ldots$ & $\mathrm{J} 171037.4-400531$ \\
\hline 3 & $17: 10: 17.76$ & $-40: 06: 12.24$ & 0.4 & $\ldots$ & $\ldots$ & $\ldots$ \\
\hline 4 & $17: 10: 00.24$ & $-40: 07: 13.08$ & 0.8 & $0498-0501194$ & $17100036-4007137$ & $\mathrm{~J} 171000.4-400714$ \\
\hline 5 & 17:10:18.00 & $-40: 08: 20.40$ & 3.0 & $0498-0501600$ & $17101812-4008233$ & $\ldots$ \\
\hline 6 & $17: 10: 22.80$ & $-40: 08: 47.40$ & 0.5 & $0498-0501699$ & $17102280-4008484$ & $\mathrm{~J} 171022.8-400847$ \\
\hline 7 & $17: 10: 11.52$ & $-40: 09: 55.08$ & 1.0 & $\ldots$ & $\ldots$ & $\ldots$ \\
\hline 8 & $17: 10: 14.88$ & $-40: 11: 31.20$ & 1.5 & $\ldots$ & $\ldots$ & $\ldots$ \\
\hline 9 & 17:09:43.44 & $-40: 13: 18.12$ & 0.6 & $0497-0499796$ & $17094361-4013188$ & J170943.6-401318 \\
\hline 10 & 17:09:33.84 & $-40: 17: 40.92$ & 0.6 & 0497-0499624 & $17093387-4017406$ & J170933.8-401740 \\
\hline 11 & 17:10:08.64 & $-40: 20: 27.60$ & 1.5 & $\ldots$ & $\ldots$ & $\mathrm{J} 171008.7-402027$ \\
\hline 12 & $17: 11: 07.68$ & $-40: 14: 13.20$ & 1.8 & $\ldots$ & $\ldots$ & J171107.9-401414 \\
\hline 13 & $17: 10: 56.88$ & $-40: 12: 16.92$ & 1.0 & $0497-0501494$ & $17105706-4012172$ & $\mathrm{~J} 171057.0-401217$ \\
\hline 14 & 17:11:01.20 & $-40: 14: 57.84$ & 1.0 & $\ldots$ & $\ldots$ & $\mathrm{J} 171101.2-401457$ \\
\hline 15 & $17: 10: 56.88$ & $-40: 20: 03.84$ & 1.2 & $\ldots$ & $\ldots$ & $\mathrm{J} 171057.0-402002$ \\
\hline 16 & $17: 10: 10.56$ & $-40: 22: 21.00$ & 5.1 & $\ldots$ & $17101009-4022164$ & $\mathrm{~J} 171010.7-402210$ \\
\hline 17 & 17:09:49.92 & $-40: 20: 22.20$ & 4.1 & $\ldots$ & 17094990-4020205 & $\ldots$ \\
\hline 18 & $17: 10: 33.36$ & $-40: 13: 08.40$ & 4.0 & $0497-0500904$ & $17103301-4013134$ & J171033.4-401318 \\
\hline 19 & $17: 10: 13.20$ & $-40: 13: 49.08$ & 1.8 & $\cdots$ & $17101317-4013527$ & $\cdots$ \\
\hline 20 & $17: 10: 23.76$ & $-40: 12: 19.08$ & 3.3 & $0497-0500702$ & $17102375-4012188$ & $\cdots$ \\
\hline 21 & $17: 10: 29.28$ & $-40: 03: 51.12$ & 1.2 & 0499-0506406 & $17102939-4003507$ & $\mathrm{~J} 171029.4-400350$ \\
\hline 22 & $17: 10: 21.60$ & $-39: 59: 49.20$ & 1.2 & $\ldots$ & $\ldots$ & $\mathrm{J} 171021.6-395950$ \\
\hline 23 & $17: 09: 42.48$ & $-39: 58: 44.04$ & 1.0 & $0500-0505315$ & $17094245-3958433$ & J170942.4-395845 \\
\hline 24 & $17: 10: 22.56$ & $-40: 07: 38.28$ & 4.0 & $0498-0501684$ & $17102235-4007330$ & $\mathrm{~J} 171022.7-400733$ \\
\hline 25 & $17: 10: 39.360$ & $-40: 12: 16.92$ & 0.9 & $\ldots$ & $\ldots$ & J171039.4-401216 \\
\hline
\end{tabular}

given the value of the parameter $p_{n}$ and is derived using

$$
\mathcal{L}=\prod \frac{1}{\sqrt{2 \pi} \sigma_{i}} \exp \left(-\frac{\chi_{i}^{2}}{2}\right)
$$

Here, $\chi_{i}$ is the chi-squared fit of our surface brightness model and our model temperature as derived when one compares these values to the $\mathrm{X}$-ray surface brightness profile and global temperature of G346.6-0.2. This is derived using $\chi_{i}^{2}=$ $\left(d_{i}-m_{i}\right) / \sigma_{i}$, where $m_{i}$ is the model prediction assuming parameter $p_{n}$ for data point $d_{i}$, and $\sigma_{i}$ is the uncertainty in $d_{i}$. Assuming that all parameters $p_{i}$ can produce a reasonable fit, we integrate $\mathcal{L}$ over the full range of parameters, producing a probability function for each parameter $p_{n}$. The $68 \%(1 \sigma)$ confidence interval for each of the parameters $p_{n}$ is derived using the minimal parameter region that encloses $68 \%$ of the integrated area under the distribution.

In Figure 5 we plot the likelihood functions for $k T_{s}, \mathcal{C}$, and $\tau$ that we obtained from our analysis. Here the shaded regions show the $1 \sigma$ confidence intervals for each parameter. In Figure 6 (left) we have plotted the model $\left(\tau, \mathcal{C}, k T_{s}\right)=$ $\left(94_{-36}^{+23}, 260_{-96}^{+63}, 0.16 \pm 0.02 \mathrm{keV}\right)$ and its uncertainty, which best reproduces the surface brightness profile (black data points) and global X-ray temperature of G346.4-0.2.

\subsection{The Inferred Properties of the Remnant}

Using these parameters, we infer the properties of the remnant using the following equations. The ISM density, $n_{\text {ism }}$, is derived using Equation (25) from White \& Long (1991), while the age of the remnant is calculated using

$$
t_{\mathrm{snr}}=\left[\frac{16 \pi \mu m_{\mathrm{H}} n_{\mathrm{ism}}}{25(\gamma+1) \kappa E_{0}}\right]^{1 / 2} R_{\mathrm{snr}}^{5 / 2} \text { year, }
$$

which is derived from Equation (8) of White \& Long (1991). Here $R_{\mathrm{snr}}$ is the radius of the remnant, $E_{0}$ is the supernova explosion energy, $\mu=0.604$ is the mean molecular weight, $m_{\mathrm{H}}$ is the mass of a hydrogen atom, $\gamma$ is the adiabatic index of the surrounding material, and $\kappa$ is the ratio of thermal and kinetic energy of the remnant as inferred from the similarity solutions of White \& Long (1991). Here, $\kappa$ is a function of $\tau$ and $\mathcal{C}$.

The explosion energy of the remnant is calculated using

$$
E_{0}=\frac{2(\gamma+1) \pi k T_{s} n_{\mathrm{ism}} R_{\mathrm{snr}}^{3}}{(\gamma-1) \kappa} \mathrm{erg} .
$$

The shock velocity is calculated using

$$
v_{s}=\frac{2}{5}\left[\frac{25(\gamma+1) \kappa E_{0}}{16 \pi \mu m_{\mathrm{H}} n_{\mathrm{ism}}}\right]^{1 / 5} t_{\mathrm{snr}}^{-3 / 5} \mathrm{~km} \mathrm{~s}^{-1} \text {, }
$$

which is derived from Equation (6) of White \& Long (1991), while the total mass of X-ray-emitting gas is derived from Equation (26) of White \& Long (1991).

In Figure 6 (right), we plot the properties of the remnant as a function of distance. Assuming a distance of $8.3 \mathrm{kpc}$, we derive a shock radius of $\sim 12 \mathrm{pc}$, an explosion energy of $\sim 7 \times 10^{50} \mathrm{erg}$, an age of $\sim 4200$ years, a shock velocity of $\sim 1120 \mathrm{~km} \mathrm{~s}^{-1}$, a gas density of the ISM just outside the shock of $\sim 0.52 \mathrm{~cm}^{-3}$, and a total swept-up mass of $\sim 53 M_{\odot}$. We find that this model $\left(\tau, \mathcal{C}, k T_{s}\right)=(94,260,0.16 \mathrm{keV})$ is able to reproduce the X-ray 
Table 4

Properties of the X-Ray Point Sources in the Field of View Detected with a Likelihood Threshold of $30 \sigma$

\begin{tabular}{|c|c|c|c|c|c|c|c|}
\hline Src & $\begin{array}{c}R_{0.2-2} \mathrm{a}^{\mathrm{a}} \\
\left(10^{-3} \mathrm{cnt} \mathrm{s}^{-1}\right)\end{array}$ & $\begin{array}{c}R_{2-10} \mathrm{~b} \\
\left(10^{-3} \mathrm{cnt} \mathrm{s}^{-1}\right)\end{array}$ & $\begin{array}{l}\text { Hardness } \\
\text { Ratio }^{c}\end{array}$ & $\begin{array}{c}N_{\mathrm{H}} \\
\left(10^{22} \mathrm{~cm}^{-2}\right)\end{array}$ & $\Gamma$ & $\chi_{\nu}^{2}(\mathrm{dof})$ & $\begin{array}{c}F_{X}^{\mathrm{d}} \\
\left(10^{-14} \mathrm{erg} \mathrm{cm}^{-2} \mathrm{~s}^{-1}\right)\end{array}$ \\
\hline 1 & $3.0 \pm 0.4$ & $3.7 \pm 0.5$ & 0.1 & $1.3_{-0.6}^{+0.9}$ & $2.2_{-0.6}^{+0.8}$ & 0.8 (14) & $5.4_{-1}^{+0.4}$ \\
\hline 2 & $4.3 \pm 0.5$ & $1.7 \pm 0.3$ & 0.5 & $0.5_{-0.4}^{+2}$ & $0.6_{-0.4}^{+0.7}$ & $1.2(14)$ & $3.4_{-0.9}^{+0.2}$ \\
\hline 3 & $0.9 \pm 0.3$ & $7.4 \pm 0.6$ & 0.8 & $1.6_{-2}^{+3}$ & $0.2_{-0.5}^{+0.6}$ & 1.1 & $3.5 \pm 0.3$ \\
\hline 4 & $3.3 \pm 0.4$ & $0.5 \pm 0.3$ & -0.7 & $0.4_{-0.4}^{+0.6}$ & $3.1_{-1}^{+2}$ & $1.0(9)$ & $1.3_{-0.1}^{+0.1}$ \\
\hline 5 & $2.2 \pm 0.4$ & $0.8 \pm 0.3$ & -0.5 & $4.0_{-2}^{+4}$ & $6.3 \pm 3$ & $2.5(8)$ & $0.9 \pm 0.5$ \\
\hline 6 & $7.2 \pm 0.6$ & $0.6 \pm 0.3$ & -0.9 & $0.01^{\mathrm{e}}$ & $2.5_{-0.3}^{+0.4}$ & 1.0 (19) & $2.5 \pm 0.2$ \\
\hline 7 & $3.1 \pm 0.4$ & $0.7 \pm 0.3$ & -0.6 & $2.3 \pm 2$ & $4.9_{-3}^{+4}$ & $1.9(8)$ & $1.4 \pm 0.7$ \\
\hline 8 & $3.7 \pm 0.4$ & $2.4 \pm 0.4$ & -0.2 & $3.4_{-1}^{+2}$ & $4.4_{-2}^{+1}$ & $0.8(15)$ & $2.7 \pm 0.3$ \\
\hline 9 & $4.8 \pm 0.5$ & $0.0 \pm 0.2$ & -1.0 & $1.0_{-0.7}^{+0.5}$ & $8.0 \pm 3$ & $0.9(10)$ & $1.8 \pm 0.4$ \\
\hline 10 & $3.1 \pm 0.4$ & $0.0 \pm 0.2$ & -1.0 & $0.3_{-0.3}^{+1}$ & $5.5 \pm 2$ & $1.34(6)$ & $2.7 \pm 1$ \\
\hline 11 & $0.0 \pm 0.2$ & $0.0 \pm 0.2$ & 0.0 & $\ldots$ & $\ldots$ & $\ldots$ & $\ldots$ \\
\hline 12 & $0.4 \pm 0.2$ & $1.5 \pm 0.3$ & 0.6 & $1.8_{-1.8}^{+7}$ & $1.2_{-1}^{+2}$ & $0.8(6)$ & $1.8 \pm 0.6$ \\
\hline 13 & $2.0 \pm 0.3$ & $0.4 \pm 0.3$ & -0.7 & $1.9_{-1.8}^{+3}$ & $5.0_{-2}^{+3}$ & $1.4(5)$ & $0.9 \pm 0.2$ \\
\hline 14 & $0 \pm 0.2$ & $1.9 \pm 0.4$ & 1.0 & $3.2_{-3}^{+9}$ & $0.7_{-1}^{+2}$ & $0.4(6)$ & $1.8 \pm 0.5$ \\
\hline 15 & $0.3 \pm 0.2$ & $0.9 \pm 0.3$ & 0.6 & $2.1_{-2}^{+12}$ & $1.8_{-1}^{+4}$ & 0.2 (4) & $2.2 \pm 0.8$ \\
\hline 16 & $0.0 \pm 0.1$ & $0.2 \pm 0.3$ & 1.0 & $\ldots$ & $\ldots$ & $\ldots$ & $\ldots$ \\
\hline 17 & $0.0 \pm 0.2$ & $0.2 \pm 0.3$ & 1.0 & $\cdots$ & $\ldots$ & $\ldots$ & $\ldots$ \\
\hline 18 & $0.8 \pm 0.3$ & $1.5 \pm 0.3$ & 0.3 & $0.6_{-0.6}^{+4}$ & $1.9_{-0.8}^{+2}$ & $1.5(6)$ & $1.1 \pm 0.4$ \\
\hline 19 & $1.4 \pm 0.3$ & $1.0 \pm 0.3$ & -0.2 & $3.3_{-2}^{+5}$ & $3.8_{-2}^{+3}$ & $1.0(6)$ & $1.3 \pm 0.3$ \\
\hline 20 & $2.5 \pm 0.4$ & $1.6 \pm 0.4$ & -0.2 & $0.8_{-0.7}^{+1}$ & $1.8_{-0.7}^{+0.9}$ & $1.5(11)$ & $2.1 \pm 0.3$ \\
\hline 21 & $1.2 \pm 0.3$ & $0.0 \pm 0.2$ & -1.0 & $1.5_{-0.9}^{+0.8}$ & $10_{-6}^{+1}$ & $1.5(3)$ & $0.4 \pm 1.1$ \\
\hline 22 & $0.7 \pm 0.3$ & $0.5 \pm 0.3$ & -0.2 & $0.01^{\mathrm{e}}$ & $1.4_{-1}^{+2}$ & 0.5 (4) & $1.6 \pm 0.1$ \\
\hline 23 & $1.2 \pm 0.3$ & $0.4 \pm 0.3$ & -0.5 & $1.6_{-0.6}^{+0.5}$ & $10_{-6}^{+1}$ & $1.6(2)$ & $0.9 \pm 0.2$ \\
\hline 24 & $2.0 \pm 0.3$ & $1.1 \pm 0.3$ & -0.3 & $2.5_{-2}^{+3}$ & $3.6_{-2}^{+3}$ & $0.3(6)$ & $1.6 \pm 0.5$ \\
\hline 25 & $0.4 \pm 0.3$ & $3.5 \pm 0.5$ & 0.80 & $20_{-18}^{+15}$ & $3.5_{-3}^{+4}$ & $1.3(9)$ & $2.0 \pm 0.7$ \\
\hline
\end{tabular}

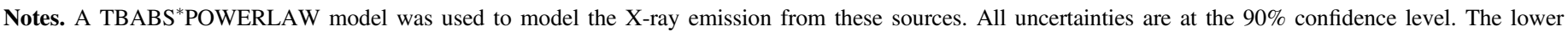
uncertainty for a number of these sources was unconstrained, due to the low number of counts, so the lower uncertainty for their fluxes is zero.

${ }^{a}$ Count rate in $0.2-2 \mathrm{keV}$ band derived from filtered $\mathrm{PN}$ data.

${ }^{\mathrm{b}}$ Count rate in $2-10 \mathrm{keV}$ band derived from filtered $\mathrm{PN}$ data.

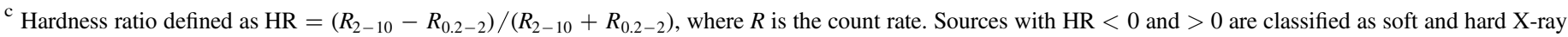
sources, respectively.

${ }^{\mathrm{d}}$ Absorbed flux in the $0.5-5.0 \mathrm{keV}$ energy band.

${ }^{\text {e }} N_{\mathrm{H}}$ for these sources is $\sim 10^{20} \mathrm{~cm}^{-2}$. We therefore fixed $N_{\mathrm{H}}$ at $0.01 \times 10^{22} \mathrm{~cm}^{-2}$.

surface brightness profile (solid magenta line in Figure 6 left) and global X-ray temperature of G346.6-0.6 quite well.

Similar to previous studies of other MM SNRs using the White \& Long (1991) model (e.g., Slane et al. 2002; Chen et al. 2004), the evaporation timescales $(\tau)$ of the cold, dense cloudlets of material inferred by the best-fit model are quite long compared to the age of the SNR $\left(>50 t_{\mathrm{snr}}\right)$. The age inferred from our study is lower than that derived by Sezer et al. (2011). These authors estimated $\sim 11 \mathrm{kyr}$ for G346.6-0.2 using the ionization timescale and electron density determined from their modeling. However, we note that they underestimated the X-ray-emitting volume of the remnant, which can lead to a larger electron density and thus a larger age.

The derived explosion energy $\left(\sim 7 \times 10^{50} \mathrm{erg}\right)$ from this model is somewhat low compared to the canonical value for SNRs $\left(\sim 10^{51} \mathrm{erg}\right)$. However, it has been shown that the White \& Long (1991) model underestimates the explosion energy of SNRs (e.g., Harrus et al. 1997).

The shock velocity inferred from this analysis is much higher than the typical velocities found in MM SNRs, which is of the order of $100 \mathrm{~km} \mathrm{~s}^{-1}$ (see, e.g., Slane et al. 2015 and references therein). In addition, the electron temperature derived from the $\mathrm{X}$-ray analysis $(k T=0.2 \mathrm{keV})$ is much lower than one would expect for a shock velocity this fast assuming $k T=(3 / 16) \mu m v_{s}^{2}$. One possible explanation for this is that the high velocity and low temperature of the thermal plasma could result if the recombining plasma originates from a fast shock that has broken through dense material and is now expanding into a low-density environment. In Section 4.2 we find that the overionized nature of the plasma most likely arises from the adiabatic cooling that is produced in this type of scenario, making it possible that these properties arise from the unique environment of the remnant.

Another possibility is that the SNR is expanding into a clumpy environment much like that presented by White \& Long (1991). As the shock passes through the dense clumpy material and into the lower-density interclump medium, this causes rapid cooling, producing an overionized plasma, while the high shock velocity could arise from the shock front traveling through the lower-density (compared to the dense cloudlets) interclump medium. However, more detailed modeling would be required to shed light on this issue, which is beyond the scope of this paper and we leave for future work.

Interestingly though, this model estimates that the amount of swept-up material is $\sim 53 M_{\odot}$, which is quite small compared to other MM SNRs (e.g., Slane et al. 2002; Auchettl et al. 2015), which have swept up $\sim 100 M_{\odot}$. However, these remnants are 

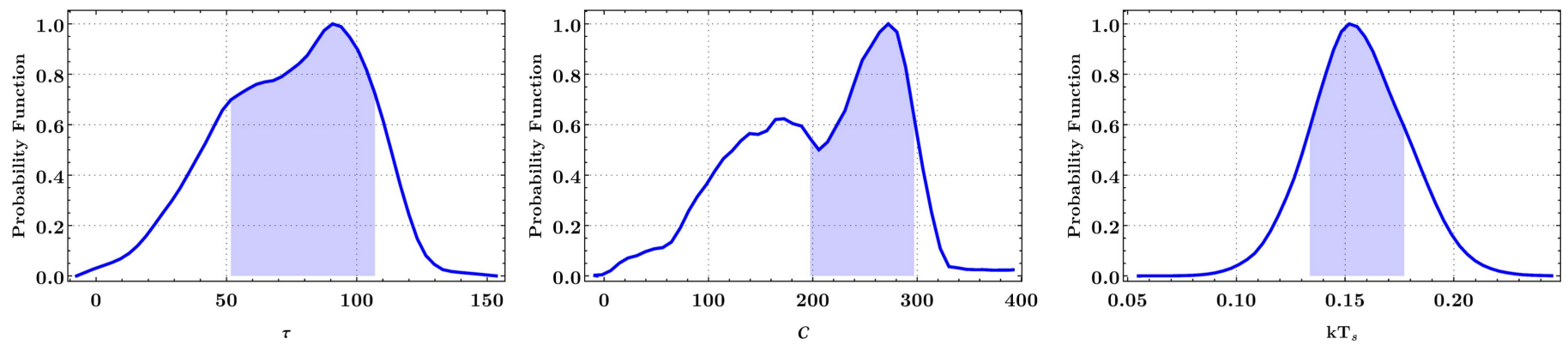

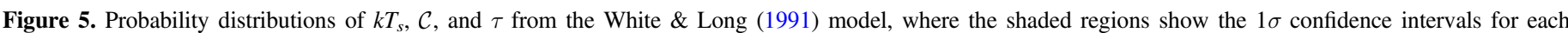
parameter. The best-fit model parameters for G346.6-0.2 are $\left(\tau, \mathcal{C}, k T_{s}\right)=\left(94_{-36}^{+23}, 260_{-96}^{+63}, 0.16 \pm 0.02 \mathrm{keV}\right)$.

usually much older, allowing them to sweep up significantly more material.

\section{Nature of the Thermal X-Ray Emission}

\subsection{Origin of the Subsolar Abundances}

Our spectral analysis indicates subsolar abundances of $\mathrm{Mg}$, $\mathrm{Si}$, and $\mathrm{S}$ in SNR G346.4-0.2, similar to the results derived using Suzaku (Sezer et al. 2011; Yamauchi et al. 2013). It was suggested that the majority of these elements are not detected because they have not yet been heated by the supernova reverse shock (Sezer et al. 2011). However, we argue that this is unlikely, since the subsolar abundances are found throughout the remnant, and the large mass of X-ray-emitting material implies that the X-ray emission arises predominantly from shocked ISM, rather than ejecta.

As Spitzer MIPS observations show evidence for a significant amount of dust associated with the remnant (Reach et al. 2006), one possible explanation for the subsolar abundances of at least $\mathrm{Mg}$ and $\mathrm{Si}$ is that these elements have condensed onto grains, lowering their gas-phase abundance. This is also supported by Spitzer IRS measurements that show strong $\mathrm{H}_{2}$ lines from the interaction of the SNR's shock with dense gas (Hewitt et al. 2009), where condensation onto dust grains is likely. However, as $\mathrm{S}$ is not a refractory element, this explanation therefore cannot explain its underabundance. As a significant nonthermal X-ray component (whose potential origin in discussed in Section 5) has also been detected in our analysis, another possibility is that the synchrotron continuum is dominating the observed X-ray spectrum. Borkowski et al. (2001) determined that a strong synchrotron continuum can cause X-ray lines to appear to be much weaker than that of a solar-abundance plasma.

\subsection{Origin of the Recombining Plasma}

A useful way to characterize the ionization state of an SNR plasma is to compare the ionization temperature $k T_{Z}$, which describes the extent that ions are stripped of their electrons, with the current electron temperature $k T_{e}$ of the plasma. Here, $k T_{e}$ is derived from the continuum, while $k T_{Z}$ is derived from line ratios (Kawasaki et al. 2002, 2005). When $k T_{Z}<k T_{e}$ or $k T_{Z}>k T_{e}$, the plasma is in an NEI state (Itoh 1977), while $k T_{Z}=k T_{e}$ implies that the plasma is in CIE. NEI states are most commonly seen in young SNRs in which shocks produce an ionizing plasma that reaches collisional equilibrium after $10^{4-5}$ years (Kawasaki et al. 2005; Smith \& Hughes 2010). However, observations of a number of MM SNRs using ASCA (Kawasaki et al. 2002, 2005), which was later confirmed by Suzaku, determined that the thermal plasma of these remnants exhibits evidence of recombination, where $k T_{Z}>k T_{e}$ (e.g., Yamaguchi et al. 2009). Evidence of rapid electron cooling in the spectra of SNRs appears in the form of a radiative recombination continuum or excess emission near the $\mathrm{K} \alpha$ lines of He-like elements. This rapid cooling can arise from either rapid cooling of electrons due to the interaction of the hot ejecta with the cold, dense surrounding environment (Cox et al. 1999), or through adiabatic expansion that can occur when the shock front of an SNR expands through a dense circumstellar material into a low-density environment (Itoh \& Masai 1989).

To determine the origin of this rapid electron cooling, we can calculate the timescale of each model. The timescale for thermal conduction is given by (Spitzer 1962)

$$
t_{\text {cond }} \sim k_{B} n_{e} l_{T}^{2} / \mathcal{K},
$$

where $l_{T}$ is the length of the thermal temperature gradient, $k_{B}$ is Boltzmann's constant, $\mathcal{K}$ is the thermal conductivity for a hydrogen plasma, and $n_{e}$ is the electron density. ${ }^{17}$ We take the length of the semimajor axis of the region we used to extract the global X-ray spectrum as the length scale, that is, $2.5 \times 10^{19} d_{8.3} \mathrm{~cm}$. Using the temperature and $n_{e}$ associated with the global X-ray spectrum, we derive the thermal conduction timescale of $\sim 500 \mathrm{kyr}$. Much like other MM SNRs, such as IC443 (Yamaguchi et al. 2009) and MSH 11 -61A (Auchettl et al. 2015), the timescale derived using thermal conduction is significantly larger than the age of the remnant we derived in Section 3, indicating that efficient thermal conduction is most likely not responsible for the rapid electron cooling.

For adiabatic cooling, the timescale can be estimated using the ionization timescale of the plasma $n_{e}=\tau_{i} t$, where $\tau_{i}$ is the ionization timescale of the plasma as derived from modeling the global X-ray spectrum. This gives us an adiabatic cooling timescale of $\sim 12 \mathrm{kyr}$, which is comparable to the age of the remnant as derived in Section 3. This implies that the origin of the recombining plasma is most likely adiabatic cooling (e.g., Lopez et al. 2013).

\footnotetext{
${ }^{17}$ To derive $n_{e}$ we use $n^{2}=4 \times 10^{14} \pi K d^{2} f^{-1} V^{-1}$, where $K$ is the normalization of our global spectrum, $f$ is the filling factor, $n_{e}=1.2 n_{\mathrm{H}}, n \approx 1.1 n_{\mathrm{H}}$ assuming Wilms et al. (2000) abundances, and $V$ is the volume of our extraction region. To derive the volume, we assume a filled ellipsoid with a semimajor radius of $3 ! 4$ (or $8.3 d_{8.3} \mathrm{pc}$ ) and a semiminor radius of 3.0 (or $7.3 d_{8.3} \mathrm{pc}$ ), which corresponds to a volume of $V=5.4 \times 10^{58} f d_{8.3}^{3} \mathrm{~cm}^{3}$.
} 

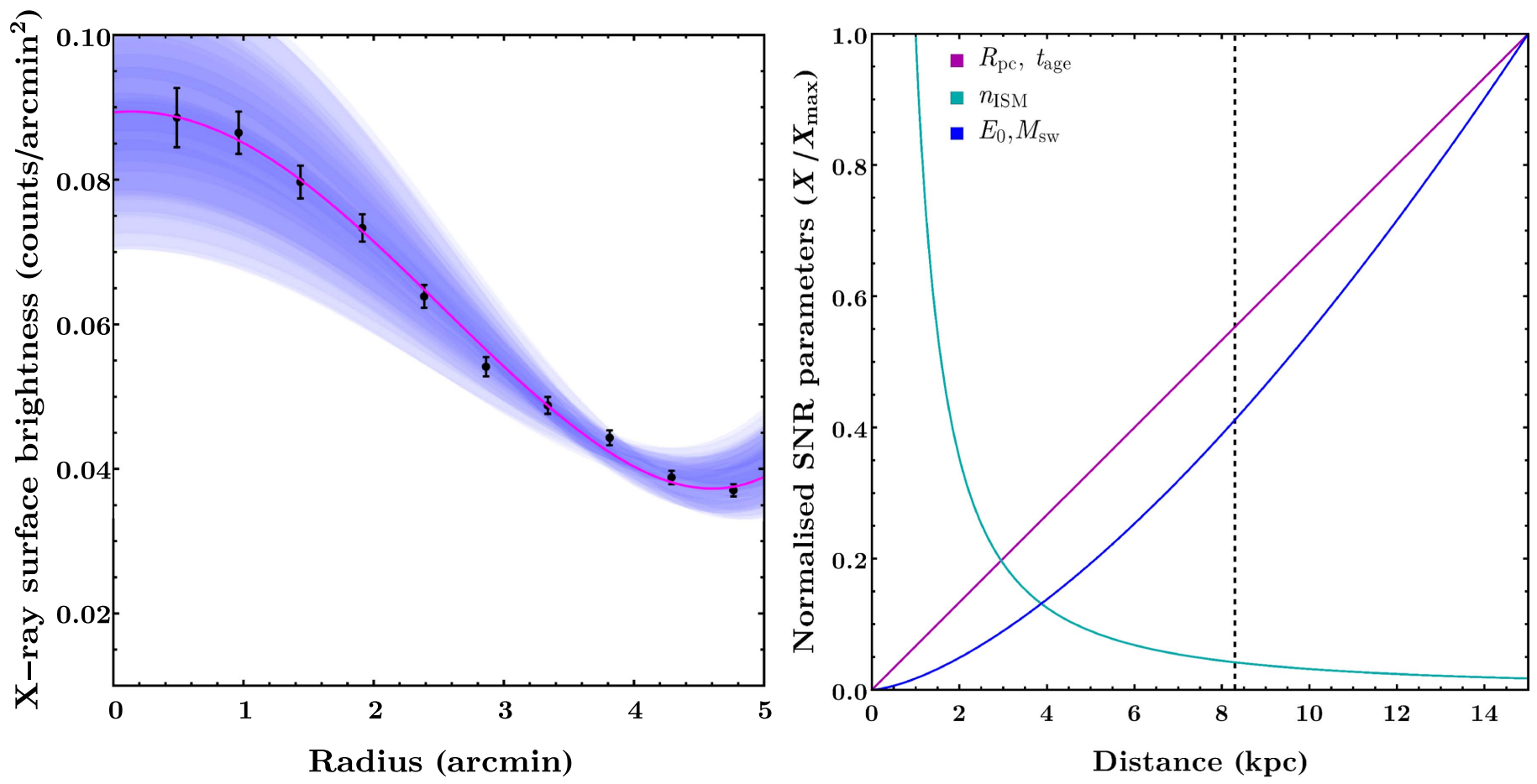

Figure 6. Left panel: comparison of the measured X-ray surface brightness of G346-0.6 with the White \& Long (1991) similarity solution that best reproduces the observed surface brightness of the remnant. Here the black data points are from the MOS1 observation of the remnant, the solid magenta line corresponds to the model using the input parameters $\left(\tau, \mathcal{C}, k T_{s}\right)=(96,260,0.16 \mathrm{keV})$, while the blue shaded region corresponds to the $68 \%$ uncertainty band for these parameters. Right panel: derived parameters for G346-0.2 using the White \& Long (1991) model. Here we plot the normalized curves for radius $\left(R_{\mathrm{pc}}\right)$, age $\left(t_{\mathrm{age}}\right)$, density of the ISM $\left(n_{\mathrm{ISM}}\right)$, explosion energy $\left(E_{0}\right)$, and swept-up mass $\left(M_{\mathrm{sw}}\right)$, where each curve is divided by $X_{\max }$, which is the maximum value of each parameter $X$ derived assuming a distance of 1-15 kpc. These are derived from Equations (2)-(4) and those referenced in the text. The $X_{\max }$ values for $R_{\mathrm{pc}}, t_{\mathrm{age}}, n_{\mathrm{ISM}}, E_{0}$, and $M_{\mathrm{sw}}$ respectively are $22 \mathrm{pc}$, $7610 \mathrm{kyr}, 13 \mathrm{~cm}^{-2}, 1.6 \times 10^{51} \mathrm{erg}$, and $1284 M_{\odot}$. The dashed black line corresponds to the values derived assuming a distance of $8.3 \mathrm{kpc}$, which is used throughout the paper.

\subsection{Supernova Type}

The supernova type of G346.6-0.2 is currently not well identified. On one hand, it was suggested that G346.6-0.2 could arise from a Type Ia SN explosion based on the relative abundance of Fe compared to Si (Sezer et al. 2011). On the other hand, Spitzer detection of spectral lines associated with shocked $\mathrm{H}_{2}$ indicates that it could be a core-collapse (CC) event (Hewitt et al. 2009; Pannuti et al. 2014). There are a number of ways to shed light on the progenitor of an SNR. This includes comparing chemical abundances such as the $\mathrm{O} / \mathrm{Fe}$ ratio to the values predicted by Type Ia and CC SN models (e.g., Iwamoto et al. 1999) since Type Ia $\mathrm{SNe}$ produce significantly more $\mathrm{Fe}$ than $\mathrm{CC} \mathrm{SNe,} \mathrm{while} \mathrm{CC}$ SNe produce a large amount of $\mathrm{O}$ compared to Type Ia SNe. One can also determine associations with nearby molecular clouds or by analyzing the asymmetry of the remnant's morphology, with $\mathrm{CC} \mathrm{SNe}$ being more asymmetric than Type Ia SNRs (Lopez et al. 2009, 2011).

Unfortunately, due to the relatively large absorption in the direction of G346.6-0.2, the poor statistics above $5 \mathrm{keV}$, and the possibility that the X-ray emission arises from shocked ISM, we are unable to use chemical abundances derived from our X-ray spectra to classify whether this remnant is a Type Ia or CC. However, we argue that G346.6-0.2 arises from a massive progenitor that underwent $\mathrm{CC} \mathrm{SN}$, based on its association with a dense molecular cloud, as well as the detection of infrared emission from shocked molecular gas (Reach et al. 2006; Hewitt et al. 2009; Andersen et al. 2011), the highly asymmetric nature of its X-ray morphology, and the fact that this remnant is located in the Galactic plane.

\section{Nature of the Hard X-Ray Tail Component}

The detection of nonthermal X-rays could originate from a number of possibilities, including an undetected pulsar or PWN, contamination from a nearby source or a population of sources, Galactic Ridge X-ray emission, or a population of relativistic particles accelerated by the SNR shock front.

\subsection{Galactic Ridge X-Ray Emission?}

G346.6-0.2 is located in the inner part of the Galactic disk, which is dominated by strong X-ray emission from the Galactic Ridge X-ray emission (GRXE). It has been shown in both deep Chandra (e.g., Ebisawa et al. 2005; Revnivtsev et al. 2009) and Suzaku (e.g., Yuasa et al. 2012; Uchiyama et al. 2013) studies that the majority of this emission can be resolved into faint Xray-emitting stellar coronae and accreting white dwarf binaries. Kaneda et al. (1997) showed that in the $0.5-10.0 \mathrm{keV}$ energy range, the GRXE is best described by an optically thin thermal plasma model with a low- and high-temperature component of $\sim 1.0 \mathrm{keV}$ and $\sim 6 \mathrm{keV}$, respectively. This was later confirmed using Chandra (e.g., Ebisawa et al. 2005) and Suzaku (e.g., Ryu et al. 2009; Yuasa et al. 2012; Uchiyama et al. 2013). Yuasa et al. (2012) attempted to decompose the high-energy $(\sim 6 \mathrm{keV})$ component of the GRXE into its various discrete source contributions. These authors found that this high-energy component is best described by a thermal plasma in CIE with a temperature of $\sim 1.2-1.7 \mathrm{keV}$ arising from coronal X-ray sources and a spectral component that arises from accreting white dwarfs with a mass $\sim 0.7 M_{\odot}$. 
The GRXE can be a major background contribution to the X-ray spectrum of diffuse sources, particularly for energies greater than $5 \mathrm{keV}$ (e.g., Yamauchi et al. 2013) or if the statistics of a source's X-ray spectrum are poor. As such, to test whether the hard X-ray tail seen in our fits arises from the hightemperature component of the GRXE, we fit the global X-ray spectrum in two ways.

First, we fit the global X-ray spectrum with an absorbed VRNEI plus an APEC model in which the temperature is set free. As discussed in more detail in Section 2.2, we find that we can produce quite a good fit (reduced $\chi^{2}=0.96$ ) to our X-ray spectrum with the temperature of the APEC model at $\sim 1.9 \mathrm{keV}$. While this value is much lower than the $6 \mathrm{keV}$ derived in previous studies (Kaneda et al. 1997; Ebisawa et al. 2005; Ryu et al. 2009), it is comparable to the softer, coronal $\mathrm{X}$-ray source contribution of the high-temperature component of the GRXE derived by Yuasa et al. (2012) or the lowtemperature component of the GRXE derived by Uchiyama et al. (2013).

Second, we fit the global X-ray spectrum using an absorbed VRNEI plus an APEC model in which we fix the temperature and abundance parameters derived by Kaneda et al. (1997) or Uchiyama et al. (2013) for the GRXE. Again, we find that this produces a similar result $\left(\chi_{r}^{2}=0.99\right)$ compared to our model listed in Tables 1 and 2. As a consequence, we cannot rule out that the additional hard component required by our fits arises from the GRXE.

Due to the relatively small size of the G346.6-0.2 (diameter $\sim 0.1$ in diameter), we do not expect the GRXE to vary significantly across the remnant considering the GRXE varies on scale heights of 0.5 latitudinally and $3^{\circ}$ longitudinally (Kaneda et al. 1997). As a consequence, we would expect that, if the GRXE is responsible for the observed hard X-ray tail seen in Figure 4, our best-fit absorbed VRNEI+POWERLAW (or VRNEI+APEC) models would produce similar $\Gamma$ (or temperatures) values. However, from Tables 1 and 2 one can see that we do see quite a large variation in the best-fit photon index (temperature) we obtain, while region 2 does not require an additional power-law or APEC component.

In addition, Yamauchi et al. (2013) carefully took into account the variation in the GRXE with scale height when modeling the X-ray spectrum from G346.6-0.2 using Suzaku by using different background regions located at different positions along their field of view for their fit. Nonetheless, they were unable to obtain a good fit with a $\chi_{r}^{2}<1.2-1.4$, implying that the spectrum might require an additional component. However, deeper observation using XMM or NUSTAR would be required to constrain the higher energy component of this remnant and to confirm or rule out the possibility of the GRXE contributing to the observed X-ray spectrum.

\subsection{Unidentified Pulsar/PWN?}

The core collapse of a massive star can lead to the formation of a neutron star. Neutron stars are rapidly rotating and have strong magnetic fields, forming a highly relativistic wind of particles. The particles in this pulsar wind interact with surrounding photon and magnetic fields, emitting both synchrotron and inverse Compton (IC) radiation, which are observed as a PWN. The emission from a PWN can be well described by a power-law spectrum with $\Gamma \sim 0.5-2.0$ (see, e.g., Gaensler \& Slane 2006), which is consistent with the photon index derived using our power-law model. Additionally, based on the global X-ray spectrum, this component has an average unabsorbed flux of $8.5 \times 10^{-13} \mathrm{erg} \mathrm{cm}^{-2} \mathrm{~s}^{-1}$ over the $0.5-7 \mathrm{keV}$ energy band. This corresponds to a luminosity of $7.3 \times 10^{33} d_{8.3}$ erg $\mathrm{s}^{-1}$, which is comparable to the observed X-ray luminosity of a large number of PWNs detected in the X-ray energy band (Kargaltsev \& Pavlov 2008).

As G346.6-0.2 most likely formed from a CC SN (see Section 4.3), it is possible that the observed nonthermal emission arises from an unidentified pulsar and its nebula. As discussed in more detail in Section 6, we search for a neutron star candidate within a circular radius defined if one assumes that the supernova explosion that created G346.6-0.2 produced a neutron star with a kick velocity of $\sim 100-500 \mathrm{~km} \mathrm{~s}^{-1}$. We find one potential candidate within the assumed distance from the remnant center (source 8 in Figure 9).

As its emission is relatively soft in nature $(\mathrm{HR}=-0.2)$, this source could potentially be a young, thermally emitting neutron star, similar to Cas A (e.g., Pavlov \& Luna 2009) or G350.1-0.3 (Lovchinsky et al. 2011). However, unlike the two cases above, our neutron star candidate exhibits some hard $(>2 \mathrm{keV}) \mathrm{X}$-ray emission. Therefore, it is possible that this source harbors an extended PWN that is responsible for the hard X-ray component detected in our analysis. By studying the exposure-corrected hard $(>3 \mathrm{keV}) \mathrm{X}$-ray image of the remnant, we find that this emission is concentrated around the position of this neutron star candidate and shows faint extended emission that extends from the position of the point source.

\subsection{Particles Accelerated by the Shock Front?}

\subsubsection{IC Scattering or Nonthermal Bremsstrahlung?}

Nonthermal X-ray emission in SNRs can arise from three main sources of emission: IC scattering, nonthermal bremsstrahlung, or synchrotron emission from shock-accelerated particles. For energies less than $10 \mathrm{keV}$ and magnetic fields between 5 and $500 \mu \mathrm{G}$, which are typically found in SNRs, IC scattering is not thought to be the dominant mechanism producing nonthermal X-ray emission in SNRs (Vink 2012). Thus we do not consider the case that IC scattering is responsible for the observed power-law component.

Nonthermal bremsstrahlung arises from nonrelativistic electrons that lose their energy via Coulomb interactions. This causes the thermalization of the low-energy tail of electrons, producing a relatively steep spectral index $(\Gamma \sim s-1<1.5$, where $s$ is the index of the electron population and $\Gamma$ is the photon index) for any nonthermal emission detected. However, Vink (2008) determined that nonthermal bremsstrahlung dominates for short ionization timescales $\left(n_{e} t \sim 10^{10} \mathrm{~cm}^{-3} \mathrm{~s}\right)$ and thus is only expected to be found in a narrow region close to the shock front. For remnants like G346.6-0.2, which have long ionization timescales, $>10^{11} \mathrm{~cm}^{-3} \mathrm{~s}$, the nonthermal bremsstrahlung component is dominated by X-ray continuum or synchrotron X-ray emission (Vink 2008, 2012). As a consequence, it is expected that the nonthermal emission detected in the soft X-ray band most likely arises from another mechanism.

\subsubsection{Synchrotron Emission?}

Another possible scenario is that the hard X-ray tail arises from synchrotron radiation produced by a population of 

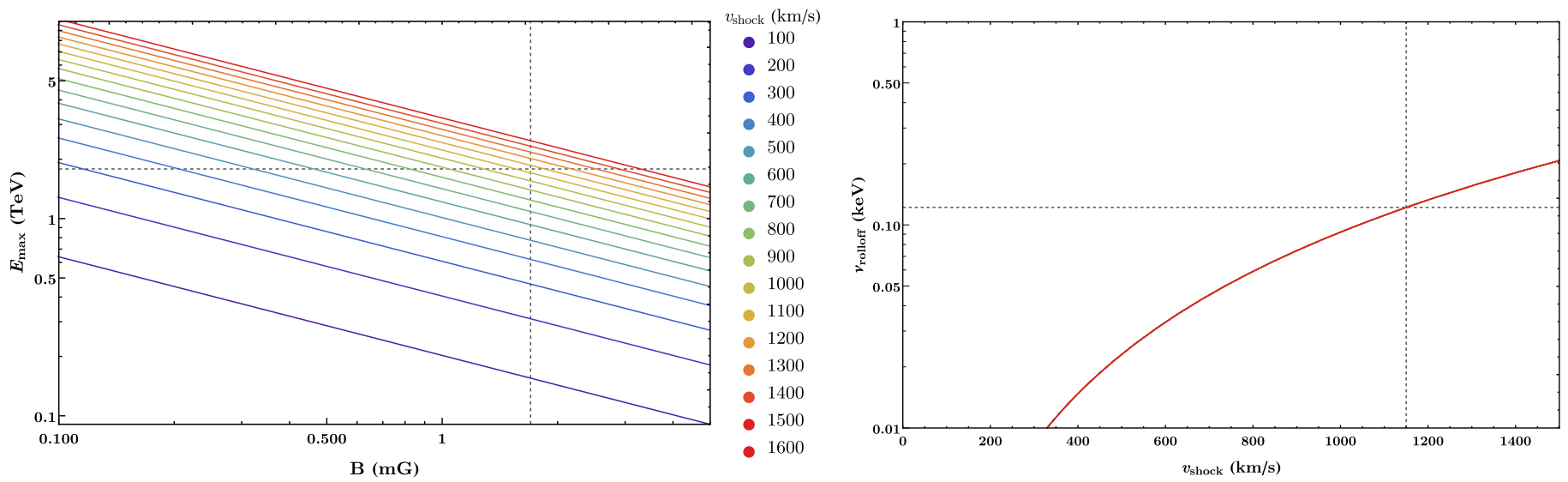

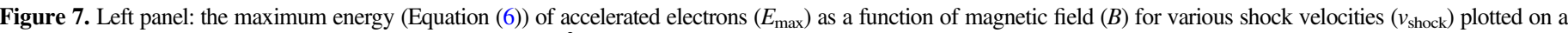

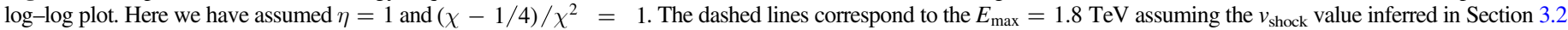

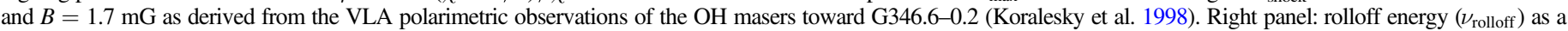
function of $v_{\text {shock }}$. The dashed lines correspond to $\nu_{\text {rolloff }}=0.12 \mathrm{keV}$ assuming the $v_{\text {shock }}$ value inferred in Section $3.2, B=1.7 \mathrm{mG}, \eta=1$ and $(\chi-1 / 4) / \chi^{2}=1$.

electrons accelerated by the SNR shock front. Nonthermal emission is usually detected in young $(<1 \mathrm{kyr})$ SNRs that have fast-moving shocks with velocities $>2000 \mathrm{~km} \mathrm{~s}^{-1}$ and magnetic fields between 50 and $250 \mu \mathrm{G}$ (Aharonian \& Atoyan 1999; Ballet 2006). Assuming that the particle acceleration is limited by synchrotron loss, the maximum energy of the underlying electron population can be estimated using (Aharonian \& Atoyan 1999; Vink 2012)

$$
\begin{aligned}
E_{\max } \sim & 32 \eta^{-\frac{1}{2}}\left(\frac{B}{100 \mu \mathrm{G}}\right)^{-\frac{1}{2}} \\
& \times\left(\frac{v_{s}}{5000 \mathrm{~km} \mathrm{~s}^{-1}}\right)\left(\frac{\chi-\frac{1}{4}}{\chi^{2}}\right)^{\frac{1}{2}} \mathrm{TeV} .
\end{aligned}
$$

Here $B$ is the magnetic field, $v_{s}$ is the shock velocity, $\eta$ is the particle acceleration efficiency, and $\chi$ is the compression ratio. Under these conditions, young SNR can accelerate electrons up to energies of 10-100 TeV (Vink 2012).

Older SNRs like MM SNRs usually have shock velocities that are effectively too slow to accelerate the electrons to the energies needed to produced X-ray synchrotron emission, assuming a magnetic field of $10-100 \mathrm{~s}$ of $\mu \mathrm{G}$. However, using VLA polarimetric observations of the $\mathrm{OH}$ masers toward G346.6-0.2, Koralesky et al. (1998) found a magnetic field of $B=1.7 \mathrm{mG}$ for G346.6-0.2 using Zeeman splitting. The lines inferred from molecular line measurements are produced from shocks being driven into cold, dense material. These conditions do not necessarily represent the properties of the X-ray-emitting region of the remnant, and thus the magnetic fields derived from these line measurements could be much higher than what is found in the bulk of the cloud material. As such, we take $B=1.7 \mathrm{mG}$ as an upper limit to the magnetic field across the remnant. This value implies that the shock velocity derived in Section 3.2 for G346 -0.2 does not need to be as high as those seen in other X-ray synchrotron SNRs (see Equation (6)).

From Equation (6) we can derive an upper limit to the maximum energy of the accelerated electrons. Assuming the shock velocity derived in Section 3.2 and the magnetic field of G346.6-0.2 derived using Zeeman splitting, the maximum energy of the underlying electron population for G346.6-0.2 is $E_{\max } \sim \eta^{-1 / 2}\left(\left(\chi-\frac{1}{4}\right) / \chi^{2}\right)^{-1 / 2} \mathrm{TeV}$. This value is slightly lower than that of other X-ray synchrotron emitting SNRs, such as 5-12 TeV for Tycho (Lopez et al. 2015) and $5 \mathrm{TeV}$ (Lazendic et al. 2004) for RX J1713.

As the nonthermal X-ray and radio emitting regions of G346.6-0.2 are not correlated (see Figure 1), it is likely that there are different electron populations producing the radio and $\mathrm{X}$-ray synchrotron emission. Thus, unlike other studies such as Reynolds \& Keohane (1999), which assume that the radio and $\mathrm{X}$-ray emission arises from the same particle population, we are unable to fit the global X-ray spectrum using the XSPEC model srcut to characterize the underlying particle energy distribution. However, even though we are unable to do this directly, assuming that the particle acceleration is limited by synchrotron loss, we can estimate an upper limit to the rolloff frequency $\nu_{\text {rolloff }}$ of the underlying particle population using (Reynolds \& Keohane 1999)

$$
\nu_{\text {rolloff }} \sim 5 \times 10^{15}\left(\frac{B}{10 \mu \mathrm{G}}\right)\left(\frac{E_{\max }}{10 \mathrm{TeV}}\right)^{2} \mathrm{keV} .
$$

Using the shock velocity inferred in Section 3.2 and $E_{\max }$ derived in Equation (6), we estimate $\nu_{\text {rolloff }} \sim 0.12 \eta^{-1 / 2}\left(\chi-\frac{1}{4}\right) / \chi^{2} \mathrm{keV}$. This is comparable to that derived by Reynolds \& Keohane (1999) for G346.6-0.6 using ASCA, as well as other SNRs with nonthermal X-ray emission such as Tycho (e.g., Hwang et al. 2002).

In addition to the simple exercise above, we also investigated how $\nu_{\text {cutoff }}$ and $E_{\max }$ of the electron population changes for different magnetic field values and shock velocities. From Figure 7 and Equation 7 one can see that as $v_{\text {shock }}$ decreases for fixed $B$-field, both $\nu_{\text {cutoff }}$ and $E_{\max }$ decrease. Similarly, if we fix $v_{\text {shock}}$, and decrease the $B$-field $E_{\max }$ decreases. Currently, known X-ray synchrotron emitting SNRs such as Tycho and SN 1006 have $E_{\max } \geqslant 1 \mathrm{TeV}$. For $v_{\text {shock }}$ inferred in Section 3.2, as $B$ decreases, $E_{\max }$ will still fall into the range seen in other SNRs that show $\mathrm{X}$-ray synchrotron emission. For a fixed $B, v_{\text {shock }}$ would have to fall below $\sim 400 \mathrm{~km} \mathrm{~s}^{-1}$ before $E_{\max } \leqslant 1 \mathrm{TeV}$. As we are unable to directly determine the cutoff energy from modeling the X-ray spectrum, and the VLA-measured $B=1.7 \mathrm{mG}$ for G346.6-0.2 represents an upper limit only, we suggest that $\nu_{\text {rolloff }}$ and $E_{\max }$ inferred from this study also represent upper limits. Further observations of this remnant to search for X-ray synchrotron filaments (e.g., Vink \& Laming 2003; Parizot et al. 2006) using 
Chandra would allow us to constrain the magnetic field, while NUSTAR observations will allow us to confirm and characterize the nonthermal emission of the remnant and thus better constrain the properties of the underlying particle population.

Compared to other X-ray synchrotron emitting SNRs such as Cas A (Gotthelf et al. 2001; Maeda et al. 2009), G1.9+0.3 (Borkowski et al. 2010), Kepler (Cassam-Chenaï et al. 2004a), RCW 86 (Broersen et al. 2014; Tsubone et al. 2017), RX J1713 (Lazendic et al. 2004; Katsuda et al. 2015), SN 1006 (Uchida et al. 2013), Tycho (Hwang et al. 2002; Eriksen et al. 2011), and Vela $\mathrm{Jr}$ (Acero et al. 2013), we find that G346.6-0.2 exhibits a thermalto-nonthermal flux ratio that is nearly an order of magnitude larger that what is seen for the other X-ray synchrotron emitting SNRs (see Figure 8). This is in contrast to the X-ray synchrotron emitting SNRs listed above that have a thermal-to-nonthermal flux ratio of $\sim 1$. If arising from particles being accelerated by the shock front of the remnant, one possible explanation for the faintness of its nonthermal component compared to its thermal component is the fact G346.6-0.2 is found in a significantly denser environment compared to other X-ray synchrotron SNRs. This could lead to significant cooling that lowers the maximum energy of the underlying electron population. As a result, fewer electrons can produce the nonthermal emission, reducing the emissivity.

Compared with other MM SNRs, only W49B is known to have shock velocities of the same order as G346.6-0.2 (Keohane et al. 2007), but as of this writing, no synchrotron emission has been detected from this remnant. All other MM SNRs have velocities in the range $50-200 \mathrm{~km} \mathrm{~s}^{-1}$, which are too slow to produce X-ray synchrotron emission, so their X-ray emission is primarily thermal in nature. ${ }^{18}$ Of the MM SNRs in which nonthermal X-ray emission has been detected, such as W28 (Zhou et al. 2014), IC443 (Bocchino \& Bykov 2001), and W44 (Frail et al. 1996), this emission arises from nonthermal bremsstrahlung or a PWN.

For SNRs found in dense environments, their shocks are usually radiative (see, e.g., Bykov et al. 2013; Slane et al. 2015), which can produce a large compression ratio leading to a lower $\nu_{\text {rolloff }}$ and $E_{\max }$. However, even if the compression ratio $\chi$ is three to four times higher than that, $\nu_{\text {rolloff }}$ can still fall within the X-ray emitting band such that G346.6-0.2 could still possibly accelerate particles to synchrotron-emitting energies. In addition, radiative shocks can also produce highly compressed magnetic fields. This can lead to significant magnetic field amplification of ISM magnetic fields, as well as enhanced cosmic-ray electron densities, which can result in strongly enhanced radio emission and to a lesser extent enhanced X-ray synchrotron radiation (see, e.g., Vink 2012 and references therein).

For the SNRs that have $\mathrm{OH}$ masers, a large fraction of these are MM SNRs. OH masers are preferentially located in regions of dense molecular material that have recently been shocked, and the magnetic fields of the MM SNRs with $\mathrm{OH}$ masers as derived from Zeeman splitting are in the range 0.2-2.2 mG (e.g., Claussen et al.

\footnotetext{
18 We note that using Suzaku, Katsuda et al. (2009) were able to fit the northwestern X-ray emission of MM SNR G156.2+5.7 using an absorbed VNEI+VNEI+POWERLAW model. They concluded that this power-law component arose from X-ray synchrotron emission from inefficient particle acceleration of a population of electrons by a shock with a velocity of $\sim 500 \mathrm{~km} \mathrm{~s}^{-1}$. However, their fits also imply that a VNEI+VNEI+NEI model is able to reproduce the observed X-ray emission in this region equally well. Uchida et al. (2012) reanalyzed the Suzaku data and found that the power-law component is more likely associated with the cosmic $\mathrm{X}$-ray background. Thus a more detailed study of this object would be required to confirm the presence (or lack) of X-ray synchrotron emission from this remnant.
}

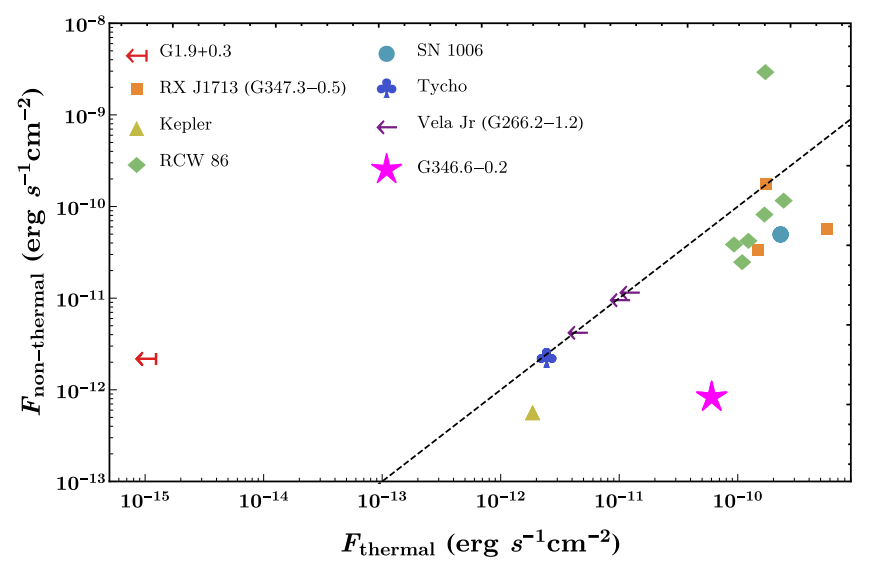

Figure 8. The unabsorbed nonthermal flux plotted against the unabsorbed thermal flux of eight well-known X-ray synchrotron emitting SNRs as derived using the best-fit models of their X-ray emission as presented in the literature. Here the fluxes are derived in the 0.5-7.0 keV energy band. For RX J1713, RCW 86, and Vela Jr, we include the flux measurements derived from multiple regions across the remnant. For G1.9+0.3 and Vela Jr, whose emission is significantly dominated by its nonthermal component, we set upper limits to their thermal X-ray emission by either following what is suggested in the literature (for G1.9+0.3) or setting the flux of their thermal emission equal to the flux of their corresponding nonthermal component (for Vela Jr). Plotted as the magenta star $(\star)$ is the unabsorbed thermal and nonthermal flux derived for G346.6-0.2 in this study. The black dashed line shows where the unabsorbed thermal and nonthermal fluxes are equal.

1997; Brogan et al. 2000, 2013). On average, this is much larger than the magnetic fields found in shell-type X-ray synchrotron emitting SNRs, which are in the range 10-100 s $\mu \mathrm{G}$ (Ballet 2006). The unique properties of G346.6-0.2 as well as the possible combination of radiative shocks and the presence of a dense environment might lead to the production of X-ray synchrotron emission in this remnant. If this power-law component is confirmed to arise from particles being accelerated by the shock front, this would make G346.6-0.2 an important new object in the class of synchrotron-emitting SNRs. However, deep observations of this source are required to confirm the origin of this component.

\section{Searching for a Neutron Star Candidate}

As we discussed in Section 4.3, G346.6-0.2 is likely a remnant of a CC SN. Detecting an associated pulsar or PWN can directly confirm this scenario. The emission from a neutron star with a PWN will be nonthermal in nature and be best described by a power-law spectrum with $\Gamma \sim 0.5-2.0$ (see, e.g., Gaensler \& Slane 2006), while for a young neutron star without a bright PWN, its emission can be described by a soft thermal component (see, e.g., Pavlov et al. 2002).

The current telescope configuration of this observation is not useful for a timing analysis to search for pulsations from a possible pulsar candidate. As a consequence, we attempted to determine the nature of the point sources in the field of view (see Figure 9) by searching for optical/IR counterparts in the B2 and R2 USNO-B1 catalogs, while extracting and modeling the $\mathrm{X}$-ray emission from each source using an extraction region with a radius of $15^{\prime \prime}$ (see Tables 3 and 4) centered on the position of the source. For all sources, particularly those that do not have an optical counterpart, we calculate the $\mathrm{HR}^{19}$ using $\left(R_{2-10}-R_{0.2-2}\right) /\left(R_{2-10}+R_{0.2-2}\right)$, where $R$ is the count rate

\footnotetext{
${ }^{19}$ Due to the relatively shallow XMM observation presented in this paper, a more detailed analysis of the point sources similar to Anderson et al. (2014) is beyond the scope of this paper.
} 


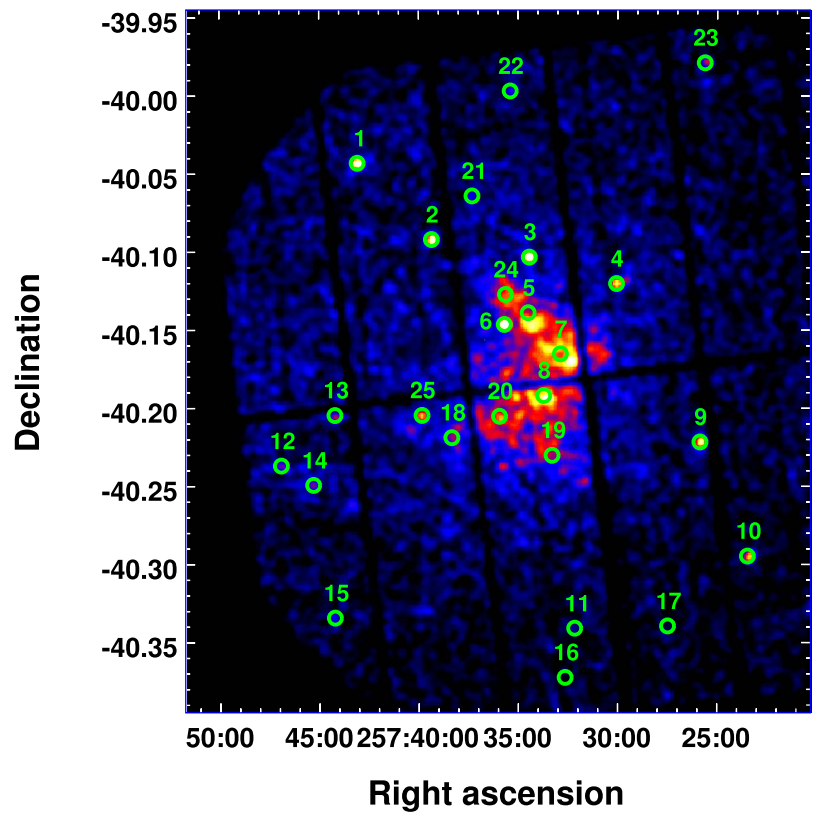

Figure 9. Point sources detected with a likelihood threshold of $30 \sigma$ or more using edetect_chain, overlaid on a $0.5-7 \mathrm{keV}$ exposure-corrected PN image of G346.6-0.2 that has been smoothed with a Gaussian of width $20^{\prime \prime}$ The sources are labeled 1-24, and their properties are listed in Tables 3 and 4.

across either the $0.2-2.0 \mathrm{keV}$ or $2.0-10.0 \mathrm{keV}$ energy bands (Misanovic et al. 2010). Sources with an HR $>0$ are hard $\mathrm{X}$-ray sources, which include objects such as a neutron star with a PWN, while those with $\mathrm{HR}<0$ are soft X-ray sources, such as stars, thermal emission from an SNR (ejecta clumps), or possibly a young neutron star without an X-ray-bright PWN.

Within the field of view, 11 out of the 25 sources detected have no optical or IR counterparts. These are good candidates for a pulsar or a PWN. Here we define a circular radius centered at $(\alpha, \delta)=\left(17^{\mathrm{h}} 10^{\mathrm{m}} 17^{\mathrm{s}},-40^{\circ} 10^{\prime} 59^{\prime \prime}\right)$ within which we would expect to find an associated neutron star given a reasonable kick velocity of $\sim 100-500 \mathrm{~km} \mathrm{~s}^{-1}$ (see, e.g., Arzoumanian et al. 2002; Hobbs et al. 2005). This corresponds to a radius of $\sim 0.2$ and 0.9 assuming a neutron star velocity of $100 \mathrm{~km} \mathrm{~s}^{-1}$ and $500 \mathrm{~km} \mathrm{~s}^{-1}$, respectively. Assuming a neutron star velocity of $100 \mathrm{~km} \mathrm{~s}^{-1}$, none of the sources we detect fall within this circular radius, while only source 8 falls within the radius expected for a neutron star traveling with a kick velocity of $500 \mathrm{~km} \mathrm{~s}^{-1}$.

Source 8 has no optical or IR counterparts and has an HR of -0.2 , indicating that the emission from this source is relatively soft and possibly thermal in nature. We were able to extract a spectrum from this source, but due to the short exposure time of our observation, the uncertainties in our fit parameters are quite large. Regardless of this, we find that the emission from source 8 can be fit using an absorbed power law with a column density of $N_{\mathrm{H}}=\left(3.4_{-1}^{+2}\right) \times 10^{22} \mathrm{~cm}^{-2}$ and a power-law index of $\Gamma=4.4_{-2}^{+1}$. The former is consistent with that of G346.6-0.2 (see Table 1), while within uncertainties either a thermal and nonthermal model ${ }^{20}$ can easily fit the observed X-ray emission. Based on its HR, it is possible that the emission from this source is more thermal in nature and could potentially arise

\footnotetext{
${ }^{20}$ Due to limited statistics, a power-law model with a steep index typically mimics the emission arising from a thermal component.
}

from a young neutron star without a bright PWN. Assuming that this point source is consistent with a neutron star, we find that the $0.5-10 \mathrm{keV}$ unabsorbed flux of this source is $\sim 9 \times 10^{-14} \mathrm{erg} \mathrm{cm}^{-2} \mathrm{~s}^{-1}$, which corresponds to an X-ray luminosity of $\sim 8 \times 10^{32} \mathrm{erg} \mathrm{s}^{-1}$ at a distance of $8.3 \mathrm{kpc}$. This is similar to the luminosities seen for other X-ray pulsars and their nebulae (see Tables 2 and 3 in Kargaltsev \& Pavlov 2008), making it not unreasonable that this is a potential neutron star candidate. This source is also coincident with the bright emission associated with region 5 (see Figure 3 ) and is consistent with both the power-law index derived from modeling and the emission from global and individual regions (Table 1), making it possible that the power-law component we observe arises from an extended PWN associated with source 8. However, deeper observations of the remnant using XMMNewton and NuSTAR will allow us to disentangle this contribution from the thermal emission of the remnant.

Apart from a young, thermally emitting neutron star, this soft X-ray point source could also potentially arise from clumps of ejecta. However, due to the low number of counts, we are unable to detect emission lines representative of ejecta emission. It is therefore difficult to differentiate between these two cases, and deeper observations of this source as well as a timing analysis would be able to shed light on the nature of this source.

\section{Conclusion}

In this paper we present XMM-Newton observations of the MM SNR G346.6-0.2. We perform imaging and spectral analysis to characterize the properties of the remnant. We find that the remnant shows bright central emission fully enclosed by the radio shell, similar to that of other MM SNRs. The X-ray emission is relatively clumpy in nature, with the bulk of the soft $\mathrm{X}$-rays found toward the north of the remnant, while the emission overlapping the position of the $\mathrm{OH}$ masers is quite hard in nature. As found in a previous Suzaku observation, we confirm that the X-ray spectrum of the SNR is well described by a cold $(0.21-0.28 \mathrm{keV})$ recombining thermal plasma with subsolar abundances of $\mathrm{Mg}, \mathrm{Si}$, and $\mathrm{S}$. But unlike some previous studies, we also find that all regions (except for region 2) require either an additional power-law component with a photon index of $\sim 2$ or a thermal APEC component with a temperature of $\sim 2.0 \mathrm{keV}$.

We investigated the possible origin of this hard X-ray tail and find that it arises either from the GRXE, an unidentified PWN, or synchrotron X-ray emission from a population of electrons accelerated by the shock front.

If this emission results from the GRXE, it is likely this hard $\mathrm{X}$-ray tail arises from thermal CIE emission arising from faint coronal X-ray sources (Yuasa et al. 2012) or from the lowtemperature component of the GRXE (see, e.g., Uchiyama et al. 2013).

Based on its morphology, its Galactic latitude, the density of the surrounding environment, and its association with a dense molecular cloud, G346.6-0.2 most likely arises from a massive progenitor that underwent $\mathrm{CC}$. As such, it is possible that this hard X-ray tail arises from an unidentified PWN. We performed a point source analysis of the sources within the field of view of the detector in an attempt to find a neutron star candidate. Defining a circular radius for which we would expect to find an associated neutron star given a reasonable kick velocity of a few $100 \mathrm{~km} \mathrm{~s}^{-1}$, we find one source (source 8) has no optical or 
IR counterparts, a photon index and HR comparable to that of young neutron stars with a PWN, and a column density similar to the remnant. However, due to the relatively short exposure time of the current $X M M$ observation, deeper observations of the source would be needed to confirm the origin of this point source.

Using the shock velocity we derived using the White \& Long (1991) model and using the magnetic field value derived from Zeeman splitting measurements as an upper limit to the magnetic field found within the remnant, it is possible that this emission could also arise from synchrotron X-ray emission from a population of electrons being accelerated by the shock front. The unique properties of this source, in addition to the possible radiative nature of its shock front and the presence of a dense environment, could possibly aid in the production of $\mathrm{X}$-ray synchrotron emission from a remnant that one would not expect to observe this type of emission. If confirmed, this would make G346.6-0.2 an important new object in the class of synchrotron-emitting SNRs.

We thank the anonymous referee for their helpful comments and suggestions that improved the quality of the paper. This work was based on observations obtained with XMM-Newton, an ESA science mission with instruments and contributions directly funded by ESA Member States and NASA.

Facility: XMM (EPIC).

Software: XMMSAS

\section{ORCID iDs}

Katie Auchettl (i) https://orcid.org/0000-0002-4449-9152

C-Y. Ng (iD https://orcid.org/0000-0002-5847-2612

Laura Lopez (iD https://orcid.org/0000-0002-1790-3148

Patrick Slane (iD https://orcid.org/0000-0002-6986-6756

\section{References}

Acero, F., Gallant, Y., Ballet, J., Renaud, M., \& Terrier, R. 2013, A\&A, 551, A7 Aharonian, F. A., \& Atoyan, A. M. 1999, A\&A, 351, 330

Anders, E., \& Grevesse, N. 1989, GeCoA, 53, 197

Andersen, M., Rho, J., Reach, W. T., Hewitt, J. W., \& Bernard, J. P. 2011, ApJ, 742, 7

Anderson, G. E., Gaensler, B. M., Kaplan, D. L., et al. 2014, ApJS, 212, 13

Arzoumanian, Z., Chernoff, D. F., \& Cordes, J. M. 2002, ApJ, 568, 289

Aschenbach, B. 1998, Natur, 396, 141

Auchettl, K., Slane, P., Castro, D., Foster, A. R., \& Smith, R. K. 2015, ApJ, 810,43

Ballet, J. 2006, AdSpR, 37, 1902

Bamba, A., Koyama, K., \& Tomida, H. 2000, PASJ, 52, 1157

Bocchino, F., \& Bykov, A. M. 2001, A\&A, 376, 248

Borkowski, K. J., Reynolds, S. P., Green, D. A., et al. 2010, ApJL, 724, L161 Borkowski, K. J., Rho, J., Reynolds, S. P., \& Dyer, K. K. 2001, ApJ, 550, 334 Broersen, S., Chiotellis, A., Vink, J., \& Bamba, A. 2014, MNRAS, 441, 3040 Brogan, C. L., Frail, D. A., Goss, W. M., \& Troland, T. H. 2000, ApJ, 537, 875 Brogan, C. L., Goss, W. M., Hunter, T. R., et al. 2013, ApJ, 771, 91

Butt, Y. M., Torres, D. F., Combi, J. A., Dame, T., \& Romero, G. E. 2001 ApJL, 562, L167

Bykov, A. M., Malkov, M. A., Raymond, J. C., Krassilchtchikov, A. M., \& Vladimirov, A. E. 2013, SSRv, 178, 599

Cassam-Chenaï, G., Decourchelle, A., Ballet, J., et al. 2004a, A\&A, 414, 545

Cassam-Chenaï, G., Decourchelle, A., Ballet, J., et al. 2004b, A\&A, 427, 199

Chen, Y., Su, Y., Slane, P. O., \& Wang, Q. D. 2004, ApJ, 616, 885

Chevalier, R. A. 1977, ARA\&A, 15, 175

Clark, D. H., Green, A. J., \& Caswell, J. L. 1975, AuJPA, 37, 75

Claussen, M. J., Frail, D. A., Goss, W. M., \& Gaume, R. A. 1997, ApJ, 489, 143

Claussen, M. J., Goss, W. M., Frail, D. A., \& Seta, M. 1999, AJ, 117, 1387
Cox, D. P., Shelton, R. L., Maciejewski, W., et al. 1999, ApJ, 524, 179

Cui, W., \& Cox, D. P. 1992, ApJ, 401, 206

Dubner, G. M., Moffett, D. A., Goss, W. M., \& Winkler, P. F. 1993, AJ, 105,2251

Dwarkadas, V. V. 2005, ApJ, 630, 892

Ebisawa, K., Tsujimoto, M., Paizis, A., et al. 2005, ApJ, 635, 214

Ergin, T., \& Ercan, E. N. 2012, in AIP Conf. Ser. 1505, 5th Int. Meeting on High Energy Gamma-Ray Astronomy, ed. A. Waldron (Melville, NY: AIP), 265

Eriksen, K. A., Hughes, J. P., Badenes, C., et al. 2011, ApJL, 728, L28

Foster, A. R., Ji, L., Smith, R. K., \& Brickhouse, N. S. 2012, ApJ, 756, 128

Frail, D. A., Giacani, E. B., Goss, W. M., \& Dubner, G. 1996, ApJL, 464, L165 Gaensler, B. M. \& Slane, P. O. 2006, ARA\&A, 44, 17

Gaensler, B. M., Slane, P. O., Gotthelf, E. V., \& Vasisht, G. 2001, ApJ, 559, 963

Gelfand, J. D., Castro, D., Slane, P. O., et al. 2013, ApJ, 777, 148

Gotthelf, E. V., Koralesky, B., Rudnick, L., et al. 2001, ApJL, 552, L39

Harrus, I. M., Hughes, J. P., Singh, K. P., Koyama, K., \& Asaoka, I. 1997, ApJ, 488,781

Hewitt, J. W., Rho, J., Andersen, M., \& Reach, W. T. 2009, ApJ, 694, 1266

Hobbs, G., Lorimer, D. R., Lyne, A. G., \& Kramer, M. 2005, MNRAS, 360,974

Huang, Y.-L., \& Thaddeus, P. 1985, ApJL, 295, L13

Hwang, U., Decourchelle, A., Holt, S. S., \& Petre, R. 2002, ApJ, 581, 1101 Itoh, H. 1977, PASJ, 29, 813

Itoh, H., \& Masai, K. 1989, MNRAS, 236, 885

Iwamoto, K., Brachwitz, F., Nomoto, K., et al. 1999, ApJS, 125, 439

Kaneda, H., Makishima, K., Yamauchi, S., et al. 1997, ApJ, 491, 638

Kargaltsev, O., \& Pavlov, G. G. 2008, in AIP Conf. Ser. 983, 40 Years of Pulsars: Millisecond Pulsars, Magnetars and More, ed. C. Bassa, Z. Wang, A. Cumming, \& V. M. Kaspi (Melville, NY: AIP), 171

Katsuda, S., Acero, F., Tominaga, N., et al. 2015, ApJ, 814, 29

Katsuda, S., Petre, R., Hwang, U., et al. 2009, PASJ, 61, S155

Kawasaki, M., Ozaki, M., Nagase, F., Inoue, H., \& Petre, R. 2005, ApJ, 631,935

Kawasaki, M. T., Ozaki, M., Nagase, F., et al. 2002, ApJ, 572, 897

Keohane, J. W., Reach, W. T., Rho, J., \& Jarrett, T. H. 2007, ApJ, 654, 938

Koralesky, B., Frail, D. A., Goss, W. M., Claussen, M. J., \& Green, A. J. 1998 AJ, 116, 1323

Koyama, K., Kinugasa, K., Matsuzaki, K., et al. 1997, PASJ, 49, L7

Koyama, K., Petre, R., Gotthelf, E. V., et al. 1995, Natur, 378, 255

Lazendic, J. S., \& Slane, P. O. 2006, ApJ, 647, 350

Lazendic, J. S., Slane, P. O., Gaensler, B. M., et al. 2004, ApJ, 602, 271

Long, K. S., Blair, W. P., Matsui, Y., \& White, R. L. 1991, ApJ, 373, 567

Lopez, L. A., Grefenstette, B. W., Reynolds, S. P., et al. 2015, ApJ, 814 132

Lopez, L. A., Pearson, S., Ramirez-Ruiz, E., et al. 2013, ApJ, 777, 145

Lopez, L. A., Ramirez-Ruiz, E., Badenes, C., et al. 2009, ApJL, 706, L106

Lopez, L. A., Ramirez-Ruiz, E., Huppenkothen, D., Badenes, C., \& Pooley, D. A. 2011, ApJ, 732, 114

Lovchinsky, I., Slane, P., Gaensler, B. M., et al. 2011, ApJ, 731, 70

Maeda, Y., Uchiyama, Y., Bamba, A., et al. 2009, PASJ, 61, 1217

Misanovic, Z., Kargaltsev, O., \& Pavlov, G. G. 2010, ApJ, 725, 931

Monet, D. G., Levine, S. E., Canzian, B., et al. 2003, AJ, 125, 984

Nobukawa, M., Uchiyama, H., Nobukawa, K. K., Yamauchi, S., \& Koyama, K. 2016, ApJ, 833, 268

Olive, K. A. \& Particle Data Group 2014, ChPhC, 38, 090001

Ozawa, M., Koyama, K., Yamaguchi, H., Masai, K., \& Tamagawa, T. 2009, ApJL, 706, L71

Pannuti, T. G., Rho, J., Heinke, C. O., \& Moffitt, W. P. 2014, AJ, 147, 55

Parizot, E., Marcowith, A., Ballet, J., \& Gallant, Y. A. 2006, A\&A, 453, 387

Pavlov, G. G., \& Luna, G. J. M. 2009, ApJ, 703, 910

Pavlov, G. G., Zavlin, V. E., \& Sanwal, D. 2002, in Neutron Stars, Pulsars, and Supernova Remnants, ed. W. Becker, H. Lesch, \& J. Trümper (New York: Academic Press), 273

Petruk, O. 2001, A\&A, 371, 267

Reach, W. T., Rho, J., Tappe, A., et al. 2006, AJ, 131, 1479

Revnivtsev, M., Sazonov, S., Churazov, E., et al. 2009, Natur, 458, 1142

Reynolds, S. P., \& Keohane, J. W. 1999, ApJ, 525, 368

Rho, J., \& Petre, R. 1998, ApJL, 503, L167

Rosen, S. R., Webb, N. A., Watson, M. G., et al. 2016, A\&A, 590, A1

Ryu, S. G., Koyama, K., Nobukawa, M., Fukuoka, R., \& Tsuru, T. G. 2009, PASJ, 61, 751

Safi-Harb, S., Petre, R., Arnaud, K. A., et al. 2000, ApJ, 545, 922

Sedov, L. I. 1959, Similarity and Dimensional Methods in Mechanics 
Sezer, A., Gök, F., Hudaverdi, M., Kimura, M., \& Ercan, E. N. 2011, MNRAS, 415,301

Skrutskie, M. F., Cutri, R. M., Stiening, R., et al. 2006, AJ, 131, 1163

Slane, P., Bykov, A., Ellison, D. C., Dubner, G., \& Castro, D. 2015, SSRv, 188,187

Slane, P., Gaensler, B. M., Dame, T. M., et al. 1999, ApJ, 525, 357

Slane, P., Hughes, J. P., Edgar, R. J., et al. 2001, ApJ, 548, 814

Slane, P., Smith, R. K., Hughes, J. P., \& Petre, R. 2002, ApJ, 564, 284

Smith, R. K., Brickhouse, N. S., Liedahl, D. A., \& Raymond, J. C. 2001, ApJL, 556, L91

Smith, R. K., \& Hughes, J. P. 2010, ApJ, 718, 583

Spitzer, L. 1962, Physics of Fully Ionized Gases (New York: Interscience)

Taylor, G. 1950, RSPSA, 201, 159

Truelove, J. K., \& McKee, C. F. 1999, ApJS, 120, 299

Truelove, J. K., \& McKee, C. F. 2000, ApJS, 128, 403

Tsubone, Y., Sawada, M., Bamba, A., Katsuda, S., \& Vink, J. 2017, ApJ, 835,34

Uchida, H., Tsunemi, H., Katsuda, S., et al. 2012, PASJ, 64, 61
Uchida, H., Yamaguchi, H., \& Koyama, K. 2013, ApJ, 771, 56

Uchiyama, H., Nobukawa, M., Tsuru, T. G., \& Koyama, K. 2013, PASJ, 65, 19

Vink, J. 2008, A\&A, 486, 837

Vink, J. 2012, A\&ARv, 20, 49

Vink, J., \& Laming, J. M. 2003, ApJ, 584, 758

Voges, W., Aschenbach, B., Boller, T., et al. 1999, A\&A, 349, 389

Warren, J. S., Hughes, J. P., Badenes, C., et al. 2005, ApJ, 634, 376

White, R. L., \& Long, K. S. 1991, ApJ, 373, 543

Whiteoak, J. B. Z., \& Green, A. J. 1996, A\&AS, 118, 329

Wilms, J., Allen, A., \& McCray, R. 2000, ApJ, 542, 914

Yamaguchi, H., Ozawa, M., Koyama, K., et al. 2009, ApJL, 705, L6

Yamauchi, S., Nobukawa, M., Koyama, K., \& Yonemori, M. 2013, PASJ, 65,6

Yamauchi, S., Shimizu, M., Nakashima, S., et al. 2014, PASJ, 66, 125

Yamauchi, S., Ueno, M., Koyama, K., \& Bamba, A. 2008, PASJ, 60, 1143

Yuasa, T., Makishima, K., \& Nakazawa, K. 2012, ApJ, 753, 129

Zhou, P., Safi-Harb, S., Chen, Y., et al. 2014, ApJ, 791, 87 